\title{
A global analysis of tonal noise in flows around aerofoils
}

\author{
Miguel Fosas de Pando ${ }^{1} \dagger$, Peter J. Schmid ${ }^{2}$ and Denis Sipp ${ }^{3}$ \\ ${ }^{1}$ LadHyX, CNRS-Ecole Polytechnique, 91128 Palaiseau, France \\ ${ }^{2}$ Department of Mathematics, Imperial College London, London SW7 2AZ, UK \\ ${ }^{3}$ ONERA/DAFE, 8 rue des Vertugadins, 92190 Meudon, France
}

(Received 5 September 2013; revised 18 March 2014; accepted 18 June 2014; first published online 30 July 2014)

The generation of discrete acoustic tones in the compressible flow around an aerofoil is addressed in this work by means of nonlinear numerical simulations and global stability analyses. The nonlinear simulations confirm the appearance of discrete tones in the acoustic spectrum and, for the chosen flow case, the global stability analyses of the mean-flow dynamics reveal that the linearized operator is stable. However, the flow response to incoming disturbances exhibits important transient growth effects that culminate in the onset of aeroacoustic feedback loops, involving instability processes on the suction- and pressure-surface boundary layers together with their cross-interaction by acoustic radiation at the trailing edge. The features of the aeroacoustic feedback loops and the appearance of discrete tones are then related to the features of the least-stable modes in the global spectrum: the spatial structure of the direct modes displays the coupled dynamics of hydrodynamic instabilities on the suction surface and in the near wake. Finally, different families of global modes will be identified and the dynamics that they represent will be discussed.

Key words: aeroacoustics, instability, separated flows

\section{Introduction}

Tonal noise on aerofoils is a type of aerodynamic noise that arises from a complex interaction of boundary-layer instabilities on the pressure and suction surfaces with the acoustic pressure field generated by the flow (see Brooks, Pope \& Marcolini 1989, for a classification of the different physical mechanisms that can lead to noise radiation on aerofoils). It is a phenomenon commonly observed on low-speed and glider aeroplanes as well as on fans and ventilators, where it manifests itself as discrete and well-defined peaks in the sound pressure spectrum approximately $30 \mathrm{~dB}$ above the background noise. Even though known and studied since the 1970s, renewed research attention in tonal noise has recently been generated owing to the increased interest in wind turbines and micro-unmanned air vehicles. In these latter applications, a minimized

$\dagger$ Present address: Departamento de Ingeniería Mecánica y Diseño Industrial, Escuela Superior de Ingeniería, Universidad de Cádiz, c/Chile 1, 11002 Cádiz, Spain. Email address for correspondence: miguel@ladhyx.polytechnique.fr 

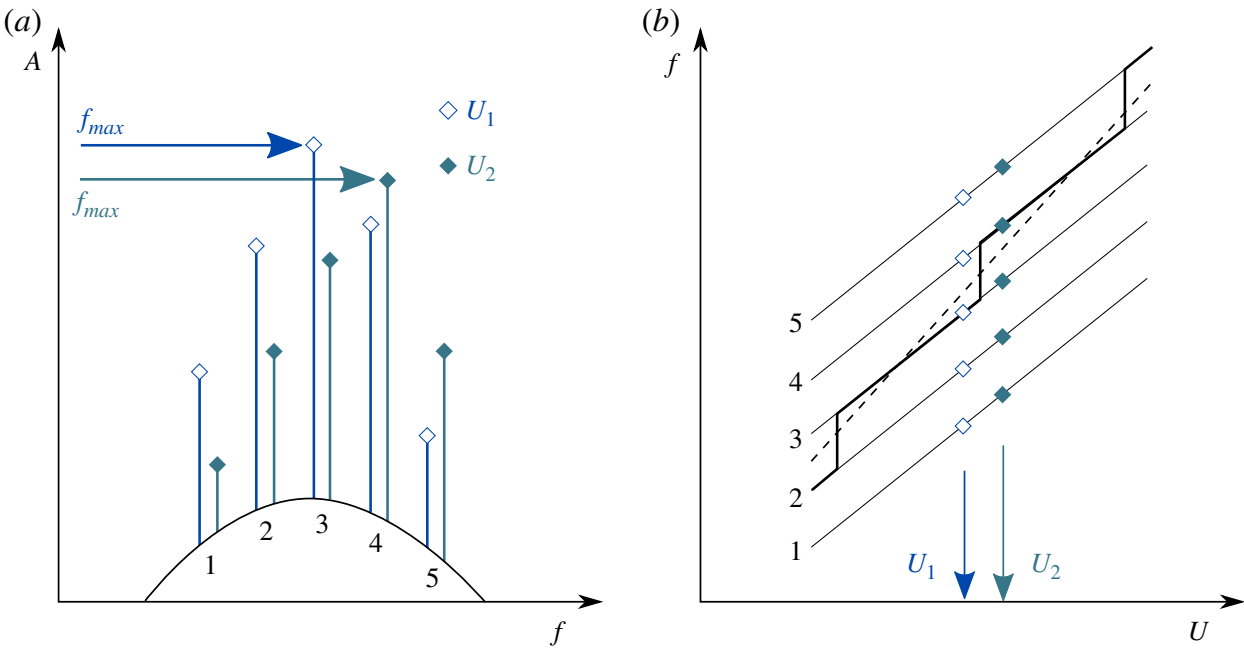

FIGURE 1. (Colour online) Sketch of typical tonal noise features: $(a)$ strong, distinct peaks in the far-field pressure spectrum rising above the broadband noise; $(b)$ dependence of the frequencies on the free-stream velocity $U$. The thick black line indicates the dominant (largest-amplitude) frequency, displaying a 'ladder' structure.

acoustic impact on the environment and a noise-free operation is desirable or even imperative.

The work of Paterson et al. (1973) is commonly considered to be the first experimental study that specifically addressed the tonal noise phenomenon on aerofoils and characterized the flow regimes where acoustic tones were present in the sound spectrum. Their work was followed by many more investigations of a theoretical (Tam 1974; Fink 1975; Kingan \& Pearse 2009), experimental (Wright 1976; Longhouse 1977; Arbey \& Bataille 1983; Brooks et al. 1989; McAlpine, Nash \& Lowson 1999; Nash, Lowson \& McAlpine 1999; Nakano, Fujisawa \& Lee 2006; Arcondoulis, Doolan \& Zander 2009) and, more recently, a numerical nature (Desquesnes, Terracol \& Sagaut 2007; Le Garrec, Gloerfelt \& Corre 2008; Sandberg et al. 2009; Jones, Sandberg \& Sandham 2010; Jones \& Sandberg 2011; Tam \& Ju 2011). However, despite four decades of research efforts and studies, an encompassing and widely accepted description of the physical mechanisms underlying the rise of discrete tones is still wanting. Based on the above-mentioned investigations, we shall give a brief résumé of the principal features and our current understanding of tonal noise on aerofoils; for a chronological presentation we refer the reader to Desquesnes et al. (2007).

Experimental studies in open-jet wind tunnels report the tonal noise phenomenon for aerofoil sections with a chord length in the range $0.08 \mathrm{~m}<c<0.3 \mathrm{~m}$, small angles of attack $0^{\circ}<\theta<8^{\circ}$, and flow speeds in the range $10 \mathrm{~m} \mathrm{~s}^{-1}<U<50 \mathrm{~m} \mathrm{~s}^{-1}$ which, for typical atmospheric conditions, translates to (chord-based) Reynold numbers in the range $10^{5}<\operatorname{Re}<2 \times 10^{6}$; see Desquesnes et al. (2007). In these cases, a pressure probe placed in the far field produces a spectrum with distinct, equispaced peaks $f_{n}$ above the broadband wind-tunnel noise (see sketch in figure $1 a$ ). These peaks are perceived by the human ear as a strong whistling sound, and pressure measurements indicate that it originates from a dipolar acoustic source located near the trailing edge. 
It has also been observed that small variations in the free-stream velocity $U$ yield shifts in the dominant frequency $\omega_{n, \max }$ that scales as $U^{0.8}$; however, at discrete velocities, previously secondary peaks $\omega_{n}$ rise and overtake the dominant frequency $\omega_{n, \max }$ in amplitude. Since the tones are discrete, the effect manifests itself as a sudden jump in the dominant frequency, resulting in a 'ladder' structure as illustrated in figure $1(b)$.

The overall trend for the maximum frequency evolution is $f_{n, \max } \sim U^{3 / 2}$ (see dashed line in figure $1 b$ ). The acoustic levels of the discrete tones increase with velocity $U$, reach a maximum, before becoming imperceptible as the boundary layers on the aerofoil surface undergo transition to turbulence. In Paterson et al. (1973), an empirical law for the overall evolution of the principal tone $f_{n, \max }$ was derived as $f=K U^{3 / 2}(c v)^{-1 / 2}$, with $K=0.011, v$ as the kinematic viscosity and $c$ as the aerofoil chord. In terms of the Reynolds number $R e$ and the Strouhal number $S t=f c / U$, the above relation reads $S t=K R e^{1 / 2}$.

The occurrence of multiple equispaced peaks following a ladder structure has been observed experimentally in several studies, among them Arbey \& Bataille (1983), Nakano et al. (2006), Takagi et al. (2006), Arcondoulis et al. (2009) and Chong \& Joseph (2012). In numerical studies, the same multiple-peak structure of the far-field pressure spectrum has been confirmed by Desquesnes et al. (2007), Le Garrec et al. (2008) and Jones \& Sandberg (2011); the frequency-ladder structure induced by a varying free-stream velocity, on the other hand, has not been investigated numerically as the calculations are computationally expensive. It is noteworthy that the experiments of Nash et al. (1999), as well as the numerical simulations of Tam \& $\mathrm{Ju}$ (2011), report only a single frequency peak. Interestingly, the experimental cases that featured multiple peaks are correlated to experiments carried out in open-jet wind tunnels, whereas the case where only one peak is observed corresponds to an experiment in a closed-section wind tunnel. In Le Garrec et al. (2008), it is suggested by means of numerical simulations that the observation of a single tone can be related to the effect of the wind-tunnel walls on the potential flow.

For common ranges of Reynolds numbers and angles of attack, owing to the adverse pressure gradient downstream of the maximum thickness of the aerofoil, the boundary layer on the pressure side detaches over the last quarter of the chord, leading to a large laminar separation bubble (Longhouse 1977; Nash et al. 1999; Desquesnes et al. 2007) that extends up to the trailing edge. Furthermore, the frequencies of the discrete tones fall within the range of frequencies at which the pressure-surface boundary layer can sustain instability waves - a fact that has been confirmed by most studies of tonal noise and by a spatial stability analysis. Nonetheless, a physical mechanism based solely on boundary-layer instabilities cannot explain a frequency-selection process with multiple discrete peaks. Despite the separation bubble on the pressure surface, the reverse flow is typically small (approximately $10 \%$ of the free-stream speed), and it is thus unlikely that absolute instabilities are present (Hammond \& Redekopp 1998). Indeed, local stability analysis of velocity profiles from experimental data (Nash et al. 1999) and numerical simulations (Jones, Sandberg \& Sandham 2008; Tam \& Ju 2011) have confirmed that the boundary layers and the wake do not sustain absolute instabilities.

Based on the above observations, different types of ad hoc mechanisms have been suggested in the last four decades, involving mostly aeroacoustic feedback loops (Tam 1974; Wright 1976; Arbey \& Bataille 1983; Desquesnes et al. 2007; Chong \& Joseph 2012). These mechanisms involve convective instabilities in the boundary layers and separated-flow regions, which amplify incoming disturbances and produce, as a result 
of the trailing-edge geometry, significant acoustic scattering. The acoustic waves that propagate into the far field would in turn excite the boundary layers upstream, thus reinforcing discrete frequency components following an integer-wavenumber phase condition.

It also appears fair to state that a proper analysis of the instability processes as well as the role of the different flow features during the tonal noise-generation process has been limited in the past by the confined scope and restrictions of classical local stability theory and the use of this tool to address non-parallel flows and long-distance feedback interactions.

The above observations are supported by the study of Jones \& Sandberg (2011). In that study the evolution of a small perturbation in aerofoil flows was investigated by means of two-dimensional numerical simulations, and therefore, their analysis was not restricted by local or parallel-flow assumptions. It was established that aeroacoustic feedback loops may be present, possibly playing a significant role in the frequency selection. It is then suggested that the tonal-noise generation mechanism is intrinsically global, as it involves instability mechanisms of different flow phenomena coupled with boundary-layer receptivity to acoustic disturbances.

In this respect, global stability theory has enabled significant progress over the past decade towards our understanding of separated flow dynamics at the onset of unsteadiness (see, for example, Gallaire, Marquillie \& Ehrenstein 2007; Ehrenstein \& Gallaire 2008; Marquet et al. 2008; Alizard, Cherubini \& Robinet 2009; Marquet et al. 2009; Cherubini, Robinet \& De Palma 2010). We thus propose a global stability analysis of the tonal-noise phenomenon as it provides a theoretical framework and basis for addressing the complex features of the problem without further simplifications or assumptions.

A great variety of flow regimes can produce discrete tones in flows around aerofoils. For the present study, we focus on a flow case similar to the one analysed in Desquesnes et al. (2007), since their two-dimensional simulations exhibited features similar to the experiments conducted by Nash et al. (1999). It should be noted that, for typical Reynolds numbers, the flow is turbulent - and therefore intrinsically three-dimensional - on a large extent of the suction surface and near the trailing edge on the pressure surface. Nevertheless, it has been found in experiments that the far-field sound radiation and associated boundary-layer instabilities are coherent in the spanwise direction, and it is thus commonly accepted that the main features of the generation of tones can be assessed by two-dimensional simulations.

Besides gaining further insight into the coupled dynamics of the boundary layers on both surfaces, the near wake and the subsequent acoustic radiation, we wish to assess whether multiple tones can be explained using global stability theory and how this type of analysis can capture the dominant observed structures as well as long-distance feedback effects.

The outline of the paper is as follows. Details about the flow configuration, the numerical scheme and the result from the nonlinear simulations are presented in $\S 2$, together with spectra from pressure measurements, properties of the mean flow and time series of the flow fields. In $\S 3$, the flow linearized about the mean flow is investigated using an impulse response analysis which reveals a sequence of dynamic components that repeat cyclically. A linear global stability analysis of the full flow is presented in $\S 4$, including an analysis of the global spectrum, the location of the associated global modes, their acoustic contributions and their role in the tonal-noise phenomenon. A summary of our results and conclusions are offered in $\S 5$. 


\section{Nonlinear simulations}

\subsection{Problem description and numerical tools}

\subsubsection{Governing equations and flow case}

The present study is concerned with uniform flow around an aerofoil section at rest in an unbounded domain. This flow obeys the two-dimensional compressible NavierStokes equations for an ideal gas; the reference values for the non-dimensionalization of the flow variables are those of the unperturbed flow (except for the entropy, where the ideal gas constant $r$ has been taken as the reference value), the reference length scale is the aerofoil chord, and the temporal scale is the residence time over one unit length. The governing equations, written in terms of the pressure $p$, the entropy $s$ and the velocity field $\boldsymbol{u}$, then read

$$
\begin{gathered}
\frac{1}{p}\left(\frac{\partial p}{\partial t}+\boldsymbol{u} \cdot \nabla p\right)=-\gamma \nabla \cdot \boldsymbol{u}+(\gamma-1)\left(\frac{\partial s}{\partial t}+\boldsymbol{u} \cdot \nabla s\right) \\
\rho\left(\frac{\partial \boldsymbol{u}}{\partial t}+\boldsymbol{u} \cdot \nabla \boldsymbol{u}\right)=-\frac{1}{\gamma M^{2}} \nabla p+\frac{1}{\operatorname{Re}} \nabla \cdot \boldsymbol{\tau}
\end{gathered}
$$

and

$$
p\left(\frac{\partial s}{\partial t}+\boldsymbol{u} \cdot \nabla s\right)=\frac{\gamma M^{2}}{\operatorname{Re}} \boldsymbol{\tau}: \nabla \boldsymbol{u}-\frac{\gamma}{(\gamma-1) \operatorname{PrRe}} \nabla \cdot \boldsymbol{q},
$$

where $R e$ stands for the Reynolds number, $\operatorname{Pr}$ for the Prandtl number, $M$ for the Mach number and $\gamma$ for the specific heat ratio. The viscous stress tensor $\tau$ and the heat flux $\boldsymbol{q}$ are given by the standard constitutive laws

$$
\boldsymbol{\tau}=\left(\nabla \boldsymbol{u}+\nabla \boldsymbol{u}^{t}\right)+\left(\frac{\mu_{v}}{\mu}-\frac{2}{3}\right)(\nabla \cdot \boldsymbol{u}) \boldsymbol{I}
$$

and

$$
\boldsymbol{q}=-\nabla T
$$

respectively. The gas has been assumed to have a constant viscosity and a constant heat conductivity. The above system of equations is augmented by the state equation $p=\rho T$, the equation for the entropy $(\gamma-1) s=\log \left(p / \rho^{\gamma}\right)$, and the definition of the speed of sound $a^{2}=p / \rho$.

In the present study, a NACA 0012 aerofoil section with a sharp trailing edge has been considered at two degrees of incidence. The modified thickness line is given by $y(x)= \pm(t / 0.2)\left(c_{0} \sqrt{x}-c_{1} x-c_{2} x^{2}+c_{3} x^{3}-c_{4} x^{4}\right)$, with $t=0.12, c_{0}=0.296375$, $c_{1}=0.12635, c_{2}=0.35195, c_{3}=0.283775$ and $c_{4}=0.10185$. We choose a Reynolds number of $2 \times 10^{5}$ and a Mach number of 0.4 . Note that the Mach number chosen for the present study is moderately higher than in the simulations performed by Desquesnes et al. (2007). This choice allows for larger time steps and thus smaller simulation times. The Prandtl number has been taken as 0.71 and the heat capacity ratio as 1.4. At the aerofoil surface, a no-slip adiabatic wall boundary condition is employed.

The above equations and boundary conditions form a closed system that can be discretized in space and integrated in time numerically, once a suitable initial condition has been specified. 


\subsubsection{Numerical schemes}

The numerical code used in the present study is based on the pseudo-characteristics formulation of Sesterhenn (2000), and the key steps of the derivation will be briefly recalled here. Prior to discretization, the compressible Navier-Stokes equations can be written in arbitrary curvilinear coordinates $\xi$ using a grid mapping given by $\boldsymbol{x}(\boldsymbol{\xi})$. The nonlinear advection terms are rearranged to explicitly contain the so-called pseudo-characteristics. In a next step, the equations are discretized in space on a structured grid using high-order compact differences. Once the sign of the advection speed for the pseudo-characteristics is known, a fifth-order upwind-biased scheme known as CULD (see Adams \& Shariff 1996) is applied. The dissipative terms and the grid metric terms are computed using a third-order centred compact scheme (see Lele 1992).

The use of a pseudo-characteristics formulation not only allows for the upwinding of advective terms but also facilitates the implementation of numerically accurate and stable boundary conditions. At the boundaries of the spatial domain, the incoming pseudo-characteristics are specified such that the flow variables exhibit the desired behaviour: no-slip adiabatic wall at the aerofoil surface, and non-reflecting inflow/outflow at the inlet/outlet of the computational domain. The reader is referred to Poinsot \& Lele (1992) and Lodato, Domingo \& Vervisch (2008) for further details on appropriate boundary conditions for compressible flow simulations.

In addition, a sponge layer has been included downstream of the aerofoil section at the outlet of the computational domain, to further attenuate spurious reflections (see Bodony 2006). The implementation has been performed by adding a spatially dependent damping term $-\sigma(\boldsymbol{x})\left(\boldsymbol{v}-\boldsymbol{v}_{\text {ref }}\right)$ to the governing equations; the variable $\boldsymbol{v}_{\text {ref }}$ denotes the reference state of the flow variables in the far field. The value of the sponge-layer parameter $\sigma(x)$ is zero for $x<L_{w}+1-L_{s}$; for $x>L_{w}+1-L_{s}$ it has been taken as

$$
\sigma(x)=A\left\{1+\exp \left(\frac{L_{s}}{x-\left(L_{w}+1\right)}+\frac{L_{s}}{x-\left(L_{w}+1-L_{s}\right)}\right)\right\}^{-1},
$$

where $A$ is a free parameter, and $L_{s}$ is the length of the sponge layer measured from the downstream outlet. The specified initial condition has been taken as the reference state $\boldsymbol{v}_{\text {ref }}$.

At this point, the temporal evolution of the compressible Navier-Stokes equations reduces to the time integration of a system of ordinary differential equations

$$
\frac{\mathrm{d} \boldsymbol{v}}{\mathrm{d} t}=\boldsymbol{F}(\boldsymbol{v})
$$

where $\boldsymbol{v}$ is the composite state vector containing the flow variables $(p, s, \boldsymbol{u})$ at every grid point, and $\boldsymbol{F}(\boldsymbol{v})$ denotes the nonlinear function that arises from the spatial discretization of the governing equations and the implementation of the boundary conditions. Finally, the above system of equations is integrated in time by means of the explicit fourth-order low-storage Runge-Kutta scheme RK4(3)5[2R+]C given in Kennedy, Carpenter \& Lewis (2000).

\subsubsection{Numerical grids and simulation parameters}

We define four numerical grids with increasing resolution and domain size: a coarse grid (G1), a refined grid (G3), an intermediate resolution (G2) and a large domain size 


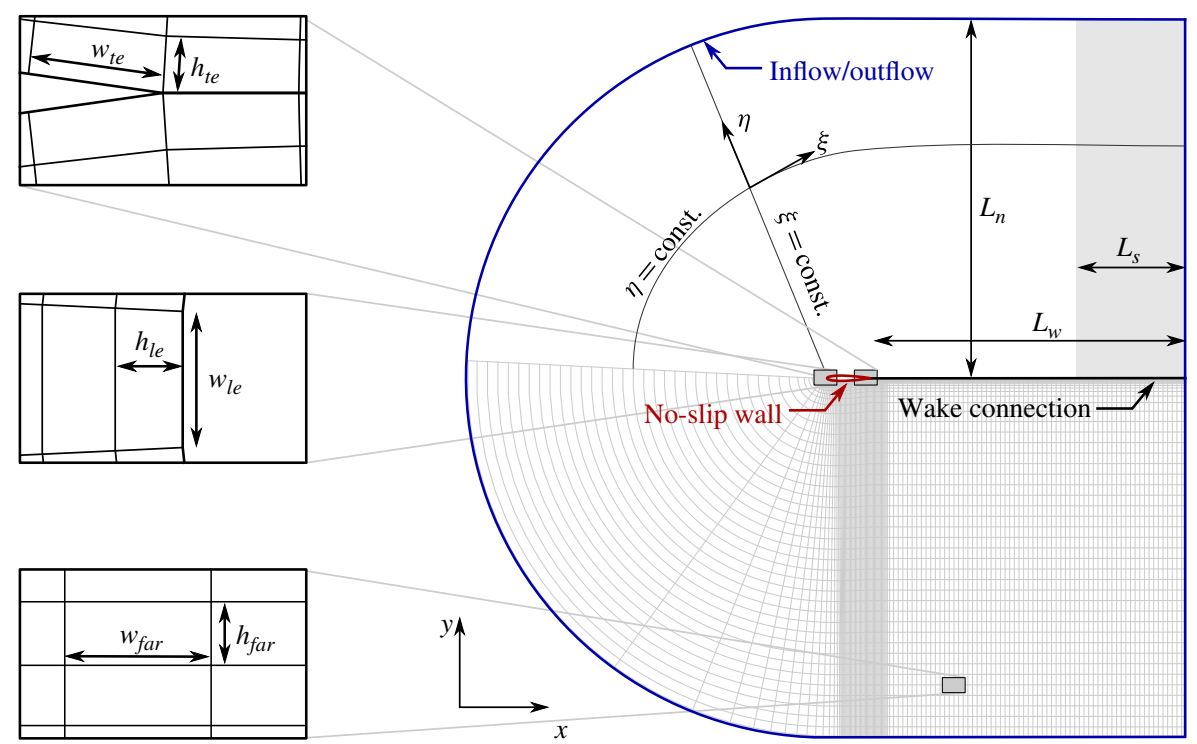

FIgURE 2. (Colour online) Numerical grid used in the present calculations. The grid geometry is defined by the length of the wake section $L_{w}$ and the normal extent $L_{n}$, and it is discretized using $m_{a}$ points on the aerofoil, $m_{w}$ points along the wake and $n$ points in the normal direction. A sponge layer of length $L_{s}$ is added at the downstream outflow boundary.

\begin{tabular}{lcccccccc}
\hline & \multicolumn{9}{c}{ Grid spacing } & & \multicolumn{2}{c}{ Domain size } \\
\cline { 2 - 5 } \cline { 5 - 6 } Simulation & $w_{\text {le }}$ & $w_{\text {te }}$ & $h_{\text {le }}=h_{\text {te }}$ & $w_{\text {far }}$ & $h_{\text {far }}$ & & $L_{w}$ & $L_{n}$ \\
G1 & $1.62 \times 10^{-3}$ & $1.50 \times 10^{-3}$ & $5.00 \times 10^{-4}$ & $6.00 \times 10^{-3}$ & $2.70 \times 10^{-2}$ & & 7.55 & 7.34 \\
G1S2 & $1.62 \times 10^{-3}$ & $1.50 \times 10^{-3}$ & $5.00 \times 10^{-4}$ & $6.00 \times 10^{-3}$ & $2.70 \times 10^{-2}$ & & 12.62 & 11.41 \\
G2 & $1.08 \times 10^{-3}$ & $1.00 \times 10^{-3}$ & $3.33 \times 10^{-4}$ & $4.00 \times 10^{-3}$ & $1.80 \times 10^{-2}$ & & 7.63 & 7.06 \\
G3 & $7.22 \times 10^{-4}$ & $6.67 \times 10^{-4}$ & $2.22 \times 10^{-4}$ & $2.67 \times 10^{-3}$ & $1.20 \times 10^{-2}$ & & 7.68 & 7.66
\end{tabular}

TABLE 1. Numerical grids and simulation parameters.

(G1S2). The computational domain and relevant geometrical parameters are illustrated in figure 2 , and the parameter values for every numerical grid are provided in tables 1 and 2 .

The computations have been run from an initial condition derived from the potential flow solution until a statistically steady state has been established. For each simulation, the initial transient dynamics, representing approximately 10 time units, have been discarded. We have stored snapshots of the evolving flow field in intervals of $\Delta t=$ 0.01, yielding a total of 15000 snapshots for case G1, and 5000 snapshots for the remaining cases.

\subsection{Instantaneous flow fields}

As a first step in our flow analysis, we use nonlinear simulations (case G3) to provide a qualitative description of the principal characteristics of tonal aerofoil noise. 

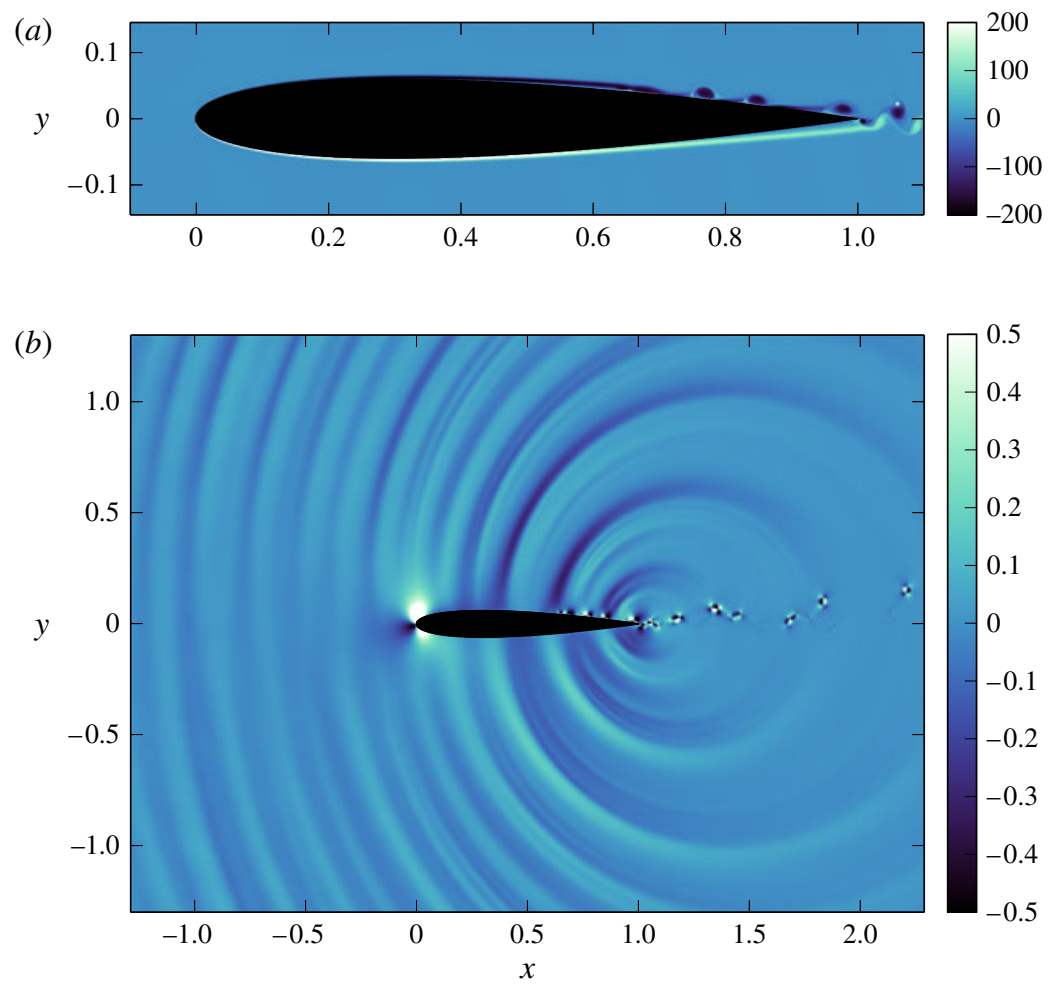

FIgURE 3. (Colour online) Instantaneous flow field showing (a) vorticity levels and (b) dilatation contours, after a quasi-periodic regime is established.

\begin{tabular}{|c|c|c|c|c|c|c|c|c|c|}
\hline \multirow[b]{2}{*}{ Simulation } & \multicolumn{3}{|c|}{ Relaxation } & \multicolumn{3}{|c|}{ Time stepping } & \multicolumn{3}{|c|}{ Spatial discretization } \\
\hline & $L_{s}$ & $A$ & $\eta^{ \pm}$ & CFL & $\Delta t$ & $T$ & $n$ & $m_{a}$ & $m_{w}$ \\
\hline G1 & 2 & 3 & 5.0 & 1.2 & $1.79 \times 10^{-4}$ & 150 & 384 & 1280 & 1280 \\
\hline G1S & 2 & 3 & 2.5 & 1.2 & $1.79 \times 10^{-4}$ & 50 & 576 & 1280 & 1920 \\
\hline $\mathrm{G} 2$ & 2 & 3 & 5.0 & 1.2 & $1.19 \times 10^{-4}$ & 50 & 512 & 1920 & 1920 \\
\hline G3 & 2 & 3 & 5.0 & 1.2 & $7.87 \times 10^{-5}$ & 50 & 768 & 2880 & 2880 \\
\hline
\end{tabular}

TABLE 2. Numerical grids and simulation parameters (continuation).

In figure 3, a typical flow field is illustrated by the instantaneous vorticity $\omega_{z}=$ $\partial_{x} v-\partial_{y} u$ and the instantaneous dilatation $d=\partial_{x} u+\partial_{y} v$ at $t=30$. The vorticity field (figure $3 a$ ) allows us to visualize boundary-layer separation with reattachment on the pressure surface and on the suction surface of the aerofoil. At the reattachment of the boundary layers, vortical structures are continuously emerging with positive and negative vorticity components. The dilatation field (figure $3 b$ ) reveals the presence of acoustic waves in the far field at a characteristic wavelength, emanating from the near wake and in counter-phase between the upper and lower half-planes of the aerofoil.

Even though the flow in this study is not strictly periodic, it is informative to visualize a representative flow-field sequence over one vortex-shedding period. The flow evolution from $t=30.00$ to $t=30.20$, shown every 0.05 time units, is depicted 


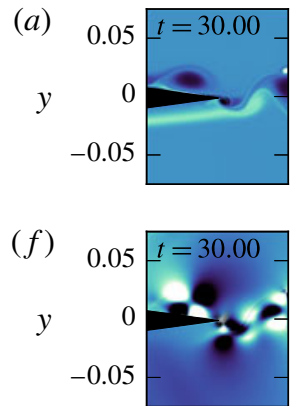

(k)

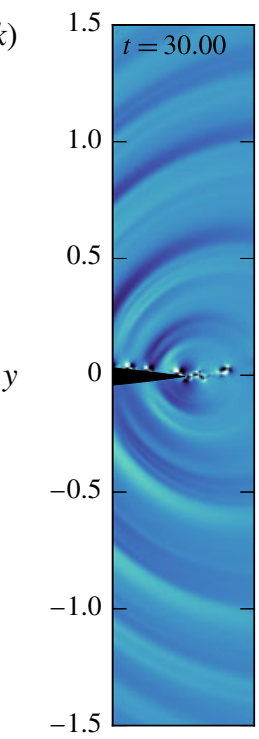

(b)

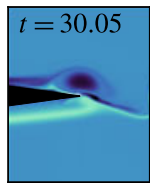

(g)

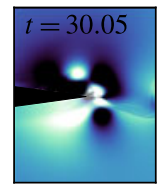

(l)

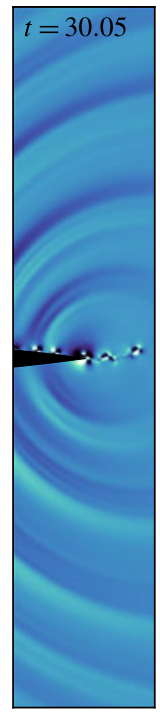

(c)

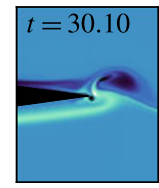

(h)

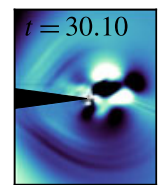

(m)

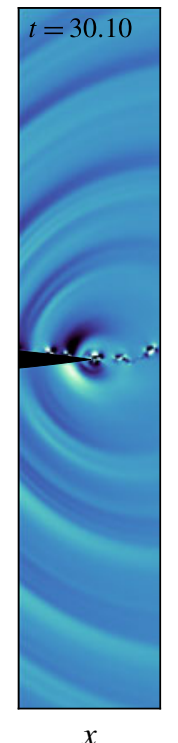

$(d)$

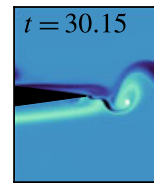

(i)

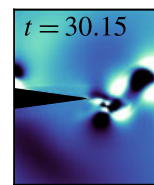

(n)

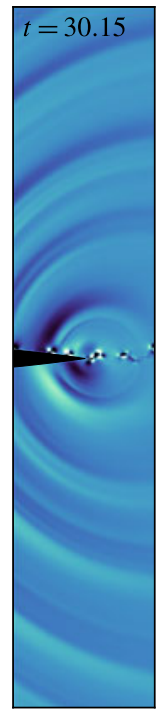

(e)

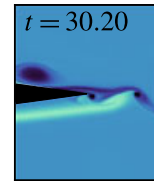

(j)

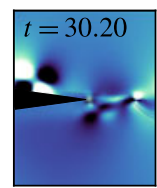

$(o)$

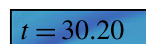

FIgURE 4. (Colour online) Representative flow field sequence showing (top row, $a-e$ ) instantaneous vorticity levels and (middle row, $f-j$ ) dilatation contours near the trailing edge, and (bottom row, $k-o$ ) dilatation contours of the far field, over one vortex shedding period. The colour scales are given in figure 3 .

in figure 4. We focus on the flow evolution near the trailing edge (top row, $a-e$ ), and middle row,$f-j$ ) and the associated far-field dynamics (bottom row, $k-o$ ).

The evolution of the vorticity field in the near wake, figure 4 (top row, $a-e$ ) shows the passage by the trailing edge of a vortex shed by the suction-surface separation bubble, and the detachment of a vortex from the separation bubble on the pressure surface. The visualization of the vorticity field illustrates that secondary vortices, with significantly smaller spatial extent, arise from the interaction of the larger vortical structures with the geometric singularity of the trailing edge.

Inspection of the dilatation field near the trailing edge, figure 4 (middle row, $f-j$ ), shows that the radiation of acoustic waves occurs at the same frequency as the shedding of vortical structures. In addition, after the formation of the secondary vortices described before, secondary acoustic waves with considerably smaller amplitude and wavelength are generated; see figure $4(c, h)$.

The far-field dilatation, figure 4 (bottom row, $k-o$ ), shows the propagation of acoustic waves emitted from the near-wake region. The analysis of the entire 

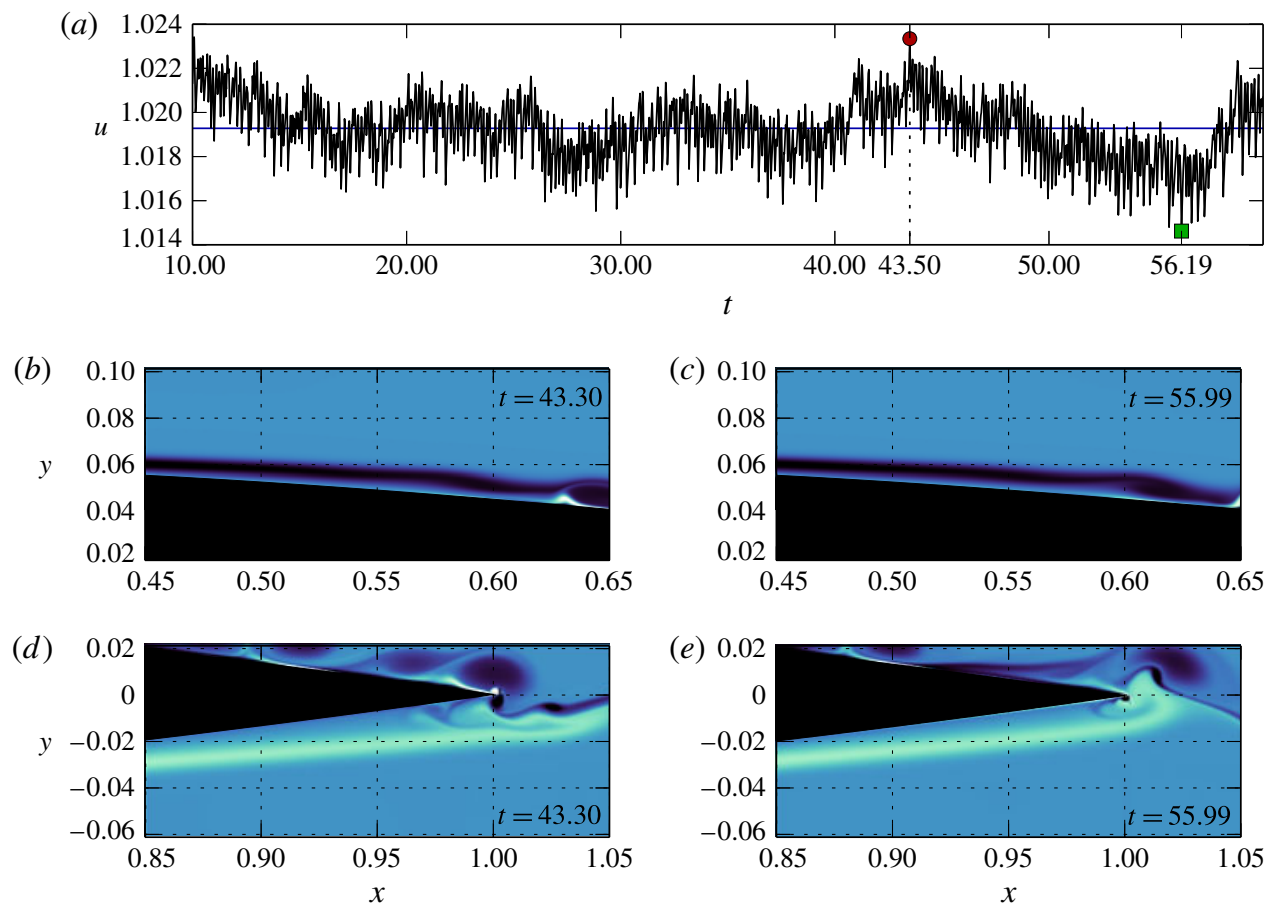

FIGURE 5. (Colour online) (a) Streamwise velocity recorded at a probe in the far field at $(x, y)=(1,0.5)$, and low-frequency flapping showing the instantaneous vorticity fields at: $(b)$ the suction-surface separation bubble at $t=43.30,(c)$ the suction-surface separation bubble at $t=55.99,(d)$ the pressure-surface separation bubble at $t=43.30$, and $(e)$ the pressure-surface separation bubble at $t=55.99$.

temporal series illustrates that the amplitude of the emitted waves depends on the precise phase relation between the passage of the vortex shed by the suction-surface separation bubble, and the shedding of a vortex from the pressure side. However, this visualization does not allow us to directly identify the contribution of these hydrodynamic features to the sound radiation.

We present in figure $5(a)$ the streamwise velocity signal recorded at the probe point $(x, y)=(1,0.5)$. In addition to the oscillations produced by the acoustic waves described above, a noticeable low-frequency oscillation is present. This feature is illustrated in figure $5(b-e)$ by displaying the vorticity fields for both separation bubbles at $t=43.30$ and $t=55.99$, the times at which the pressure signal maximum and minimum is reached, taking into account the retarded time between the trailing edge and the probe point. The time at which the streamwise velocity signal reaches a maximum or minimum is correlated with a low-frequency motion in the pressure-surface separation bubble; no substantial changes have been observed in the separation bubble on the suction surface.

These low-frequency oscillations seem to have been overlooked in previous calculations and experiments that address the tonal-noise generation mechanism; nonetheless, it is a well-known feature of elongated separation bubbles (see Dovgal, Kozlov \& Michalke 1994, and references therein). 


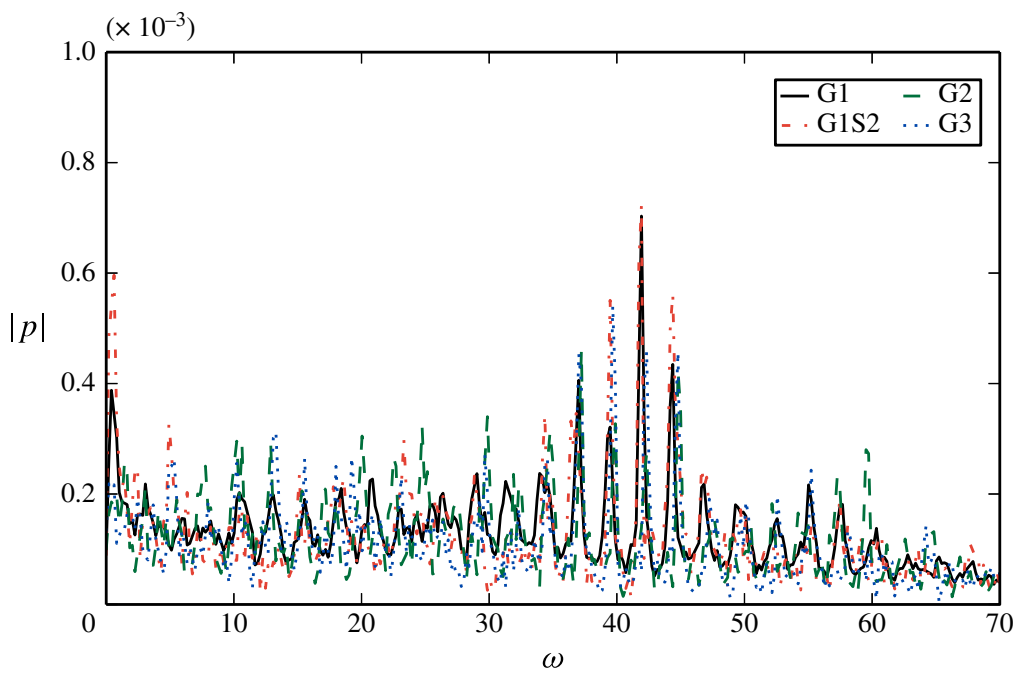

FIGURE 6. (Colour online) Frequency content of the pressure signal extracted from the probe at $(x, y)=(1,0.5)$ for different numerical grids.

\begin{tabular}{|c|c|c|c|c|c|c|c|c|}
\hline \multirow[b]{3}{*}{ Simulation } & \multicolumn{8}{|c|}{ Frequency bands } \\
\hline & \multicolumn{2}{|c|}{$35.5-38.1$} & \multicolumn{2}{|c|}{$38.1-40.8$} & \multicolumn{2}{|c|}{$40.8-43.4$} & \multicolumn{2}{|c|}{$43.4-46.1$} \\
\hline & $\omega_{\text {peak }}$ & $|p|_{\text {peak }}$ & $\omega_{\text {peak }}$ & $|p|_{\text {peak }}$ & $\omega_{\text {peak }}$ & $|p|_{\text {peak }}$ & $\omega_{\text {peak }}$ & $|p|_{\text {peak }}$ \\
\hline G1 & 37.02 & $4.1 \times 10^{-4}$ & 39.47 & $3.2 \times 10^{-4}$ & 41.93 & $7.0 \times 10^{-4}$ & 44.38 & $4.3 \times 10^{-4}$ \\
\hline G1S2 & 36.82 & $3.5 \times 10^{-4}$ & 39.47 & $5.5 \times 10^{-4}$ & 41.93 & $7.2 \times 10^{-4}$ & 44.38 & $5.6 \times 10^{-4}$ \\
\hline $\mathrm{G} 2$ & 37.22 & $4.6 \times 10^{-4}$ & 39.88 & $3.3 \times 10^{-4}$ & 42.34 & $2.7 \times 10^{-4}$ & 44.79 & $4.2 \times 10^{-4}$ \\
\hline G3 & 37.02 & $4.5 \times 10^{-4}$ & 39.68 & $5.4 \times 10^{-4}$ & 42.34 & $4.6 \times 10^{-4}$ & 44.79 & $4.6 \times 10^{-4}$ \\
\hline
\end{tabular}

TABLE 3. Values of selected frequency components and their amplitudes in the pressure spectrum shown in figure 6.

\subsection{Acoustic spectrum}

For each numerical grid, the frequency content of the pressure signal extracted at $(x, y)=(1,0.5)$ has been estimated using the Welch method, together with a Hamming window; the window length has been taken as 3072 samples with an overlap of $50 \%$. The amplitude of the pressure signal for varying frequency is given in figure 6.

A set of equally spaced peaks is easily identified in the pressure spectrum in the frequency range $35<\omega<45$. These discrete components are clearly related to the acoustic emission observed in the flow evolution; see figure 3. The precise values of the frequencies and amplitudes of the acoustic tones are included in table 3.

Even though the amplitudes of the acoustic tones cannot be considered fully converged, the presence of the tones and the frequency at which they occur are independent of the domain size and grid resolution. Low-frequency oscillations $(\omega<2)$ are also readily identified in the frequency spectrum.

\subsection{Mean-flow analysis}

Before launching into a global analysis, the characteristics of the mean flow will be described. The pressure coefficient distribution over the aerofoil, not shown here, 


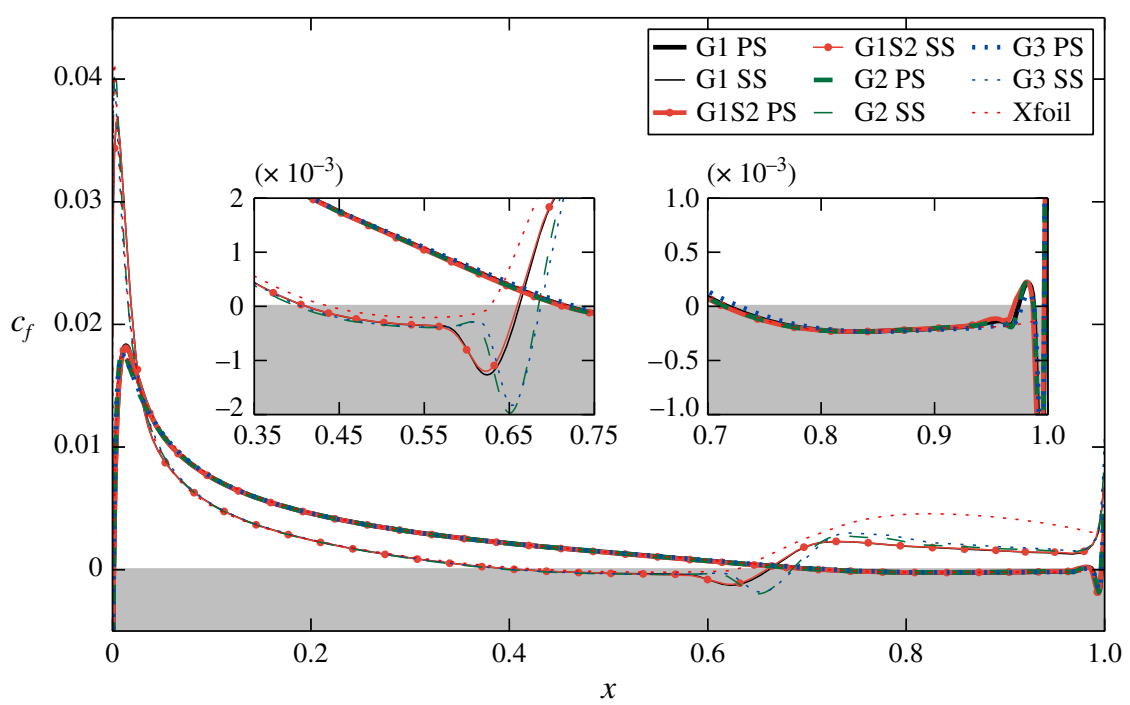

FIgURE 7. (Colour online) Mean-flow skin-friction coefficient distribution over the aerofoil. SS and PS denote suction surface and pressure surface respectively.

\begin{tabular}{lccccccc} 
& \multicolumn{3}{c}{ Pressure surface } & & \multicolumn{3}{c}{ Suction surface } \\
\cline { 2 - 4 } \cline { 6 - 8 } Simulation & $x_{s}$ & $x_{r}$ & $u_{\min }$ & & $x_{s}$ & $x_{r}$ & $u_{\min }$ \\
G1 & 0.717 & 0.974 & -0.11 & & 0.411 & 0.662 & 0.05 \\
G1S2 & 0.714 & 0.970 & -0.12 & & 0.411 & 0.659 & -0.04 \\
G2 & 0.715 & 0.974 & -0.11 & & 0.400 & 0.684 & -0.06 \\
G3 & 0.728 & 0.977 & -0.10 & & 0.405 & 0.686 & -0.07
\end{tabular}

TABLE 4. Separation, reattachment points and maximum reverse flow on the pressure-surface and suction-surface boundary layers.

confirms that the boundary layers on both surfaces of the aerofoil are subjected to adverse pressure gradients: from $x=0.04$ to the trailing edge for the suction surface, and from $x=0.24$ to the trailing edge for the pressure surface. The separation point and the zones of reverse flow can further be determined by the zero crossing and negative values, respectively, of the skin-friction coefficient $c_{f}(x)=2 \operatorname{Re}^{-1}(\boldsymbol{t} \cdot \boldsymbol{\tau} \cdot \boldsymbol{n})$. In this expression, $\boldsymbol{n}$ is the wall-normal vector, and $\boldsymbol{t}$ is the vector tangential to the aerofoil surface in the direction of the free-stream velocity. The skin-friction coefficient, shown in figure 7, confirms the presence of separation bubbles on the pressure and on the suction surfaces. The mean separation and reattachment points, together with the maximum reverse flow are given in table 4 . Note that, in the case studied by Desquesnes et al. (2007) where tonal noise is present, no suction-surface separation was observed, but marginal separation was found instead. Given the parameter choice in both cases, it is conceivable that this discrepancy arises from a different value of the Mach number.

The boundary-layer velocity profiles are presented in figure 8 showing the walltangential velocity component $u_{t}(n, x)$. The velocity profiles on the suction surface (figure $8 a$ ) and on the pressure surface (figure $8 b$ ) are characterized by inflection points due to the combined effect of the negative pressure gradients and the separation 

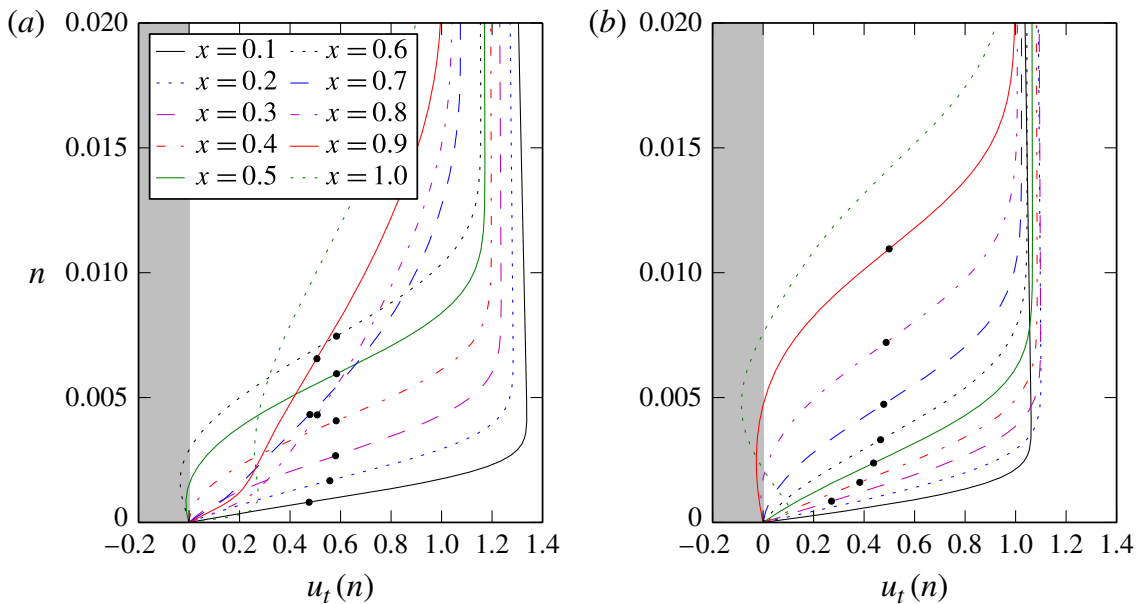

FIGURE 8. (Colour online) Boundary layer mean velocity profiles on $(a)$ the suction surface and $(b)$ the pressure surface. The inflection points are indicated in black.

bubbles, thus indicating that hydrodynamic instabilities may grow by inviscid mechanisms. These observations are in agreement with the results of Nash et al. (1999) and Desquesnes et al. (2007). Local stability characteristics of typical velocity profiles found in separated boundary-layer flows are given in Dovgal et al. (1994).

Previous studies - experimental and numerical - have drawn attention to the standard deviation of the mean flow in the separation bubbles, which for the present calculations is shown in figure 9. The standard-deviation profiles on the suction side consist of two peaks that appear after the mean reattachment point, indicating an increased amount of unsteadiness caused by vortex growth and advection. On the pressure surface, only a single peak in the standard deviation appears at the separated shear layer. By inspection of the averaged streamlines close to the trailing edge (see figure 9, inset), we observe that the pressure-surface separation bubble gives rise to two counter-rotating trailing vortices that continue into the wake. This feature is related to the two-dimensional nature of the simulation.

At this point, the presented calculations and observations suggest that a strong interaction occurs between (i) the dynamics of the separated flow on both sides, (ii) vortices shedding into the wake and (iii) the scattered acoustic waves. Our aim is then to understand the different degrees of participation of these flow features in the generation of sound and their role in the establishment of a self-sustained process. These goals will be addressed in the following sections.

\section{Impulse response analysis}

\subsection{Numerical set-up}

Even though most global stability concepts are formally defined in the continuous domain, we directly present the framework for the spatially discretized equations. Once the nonlinear compressible Navier-Stokes equations have been spatially discretized, the calculation of the temporal evolution is reduced to the integration of a set of ordinary differential equations given in (2.4). Linear analysis is then based on the Jacobian of this nonlinear function $\boldsymbol{F}(\boldsymbol{v})$ around a base flow $\boldsymbol{V}$. 


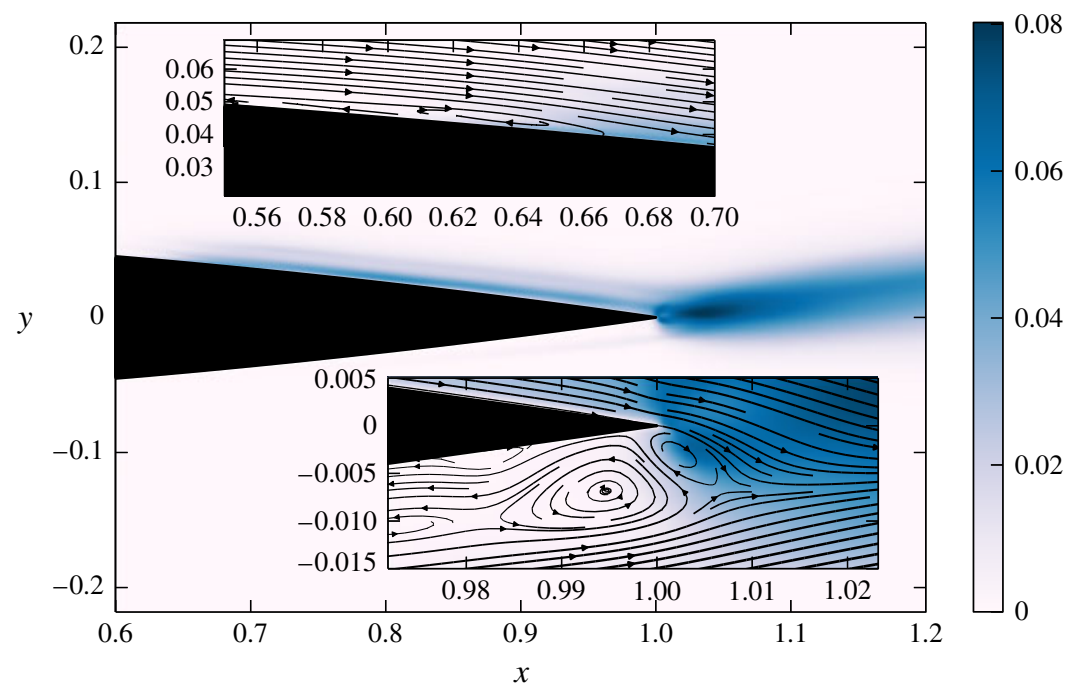

FIgURE 9. (Colour online) Root-mean-square (r.m.s.) of velocity fluctuations, showing streamlines in the regions of growth of unsteadiness near the reattachment of the separation bubbles on the suction surface and on the pressure surface.

Provided that the physical mechanisms under investigation are well represented by the nonlinear model and its linearized dynamics, the operator $\boldsymbol{A}$ will form the foundation for our further study. In the following, we consider the linear dynamics of a small perturbation $\boldsymbol{u}$ given by the initial-value problem

$$
\frac{\mathrm{d} \boldsymbol{v}}{\mathrm{d} t}=\boldsymbol{A} \boldsymbol{v}, \quad \text { with } \boldsymbol{A}=\left(\frac{\partial \boldsymbol{F}}{\partial \boldsymbol{v}}\right)_{\boldsymbol{V}} \quad \text { and } \quad \boldsymbol{v}(0)=\boldsymbol{u} .
$$

\subsubsection{Choice of base flow}

The Reynolds number of the first bifurcation for our aerofoil flow occurs at approximately $R e_{c} \approx 9 \times 10^{3}$ (Akbar 2010); the steady solution for the present flow configuration, not shown here, displays boundary-layer detachment close to the aerofoil nose, leading to a massive separation bubble that extends over dozens of chord lengths downstream. A similar observation was made in the numerical study of Jones \& Sandberg (2011), where the steady solution was deemed unphysical, and the mean-flow dynamics were considered.

Previous studies on more simplified geometries have observed that a stability analysis of the mean flow can accurately reproduce the frequencies found in fully nonlinear simulations (see Barkley 2006, for example). In the context of tonal noise, previous investigations have highlighted the predominant role of the stability characteristics of the pressure-side boundary-layer, which is not affected by nonlinear saturation effects. Therefore, the mean flow will be taken as the linearization point (referred to as the base flow) for the global stability analysis.

\subsubsection{Evaluation of the linearized operator}

Although conceptually simple, the linear algebra problems that arise from linear stability calculations are not straightforward to solve: the high dimensionality of $\boldsymbol{A}$ 
puts severe restrictions on the numerical methods that can be chosen for practical implementations. This choice is further restricted by the fact that the operator $\boldsymbol{A}$ is in general dense, owing to our choice of discretization. The limitation is twofold: the storage required for the full operators is prohibitive large, and the computational time required for solving the entire problem using dense algorithms far exceeds typical computational resources.

In this context, the only numerical methods that provide a solution to our problem are necessarily based on iterative matrix-free algorithms. Algorithms based on Krylov subspace techniques are thus the methods of choice, as they solely rely on matrix-vector products $\boldsymbol{A} \boldsymbol{v}$. For our case, we have augmented our direct numerical simulation code by an evaluation procedure of matrix-vector products for the linearized operator $\boldsymbol{A}$ (see Fosas de Pando, Sipp \& Schmid 2012). At this point, the above initial-value problem can been integrated using an exponential Krylov time-integration scheme (Sidje 1998).

\subsection{Temporal evolution of the norm}

In the following, the initial condition $\boldsymbol{u}$ is taken as a divergence-free localized perturbation at the leading edge of the aerofoil, where the vector components are defined by

$$
\begin{aligned}
p(x, y) & =0, \\
s(x, y) & =0, \\
\bar{\rho}(x, y) u(x, y) & =-\left(y-y_{0}\right) / r_{0} \exp \left(-\left(\left(x-x_{0}\right)^{2}+\left(y-y_{0}\right)^{2}\right) / r_{0}^{2}\right), \\
\bar{\rho}(x, y) v(x, y) & =\left(x-x_{0}\right) / r_{0} \exp \left(-\left(\left(x-x_{0}\right)^{2}+\left(y-y_{0}\right)^{2}\right) / r_{0}^{2}\right),
\end{aligned}
$$

where $x_{0}=-0.015, y_{0}=-0.1, r_{0}=0.005$ and $\bar{\rho}(x, y)$ is the non-dimensional base-flow density.

The subsequent flow evolution has been computed over $T=30$ time units, and snapshots of the flow field have been collected every $\Delta t=0.01$. In figure 10, we present the evolution of the norm (details about the norm used in this work are given in appendix A) of the perturbation $\|\boldsymbol{v}(t)\|=\left\|\mathrm{e}^{t A} \boldsymbol{u}\right\|$ as a function of time. It is important to point out that only the contributions of perturbations within a circle of radius 1.25 centred at mid-chord are considered. Formally, the above measure is a semi-norm, and it has been chosen such that the features described below become independent of the domain size. Two regimes can clearly be distinguished: (i) for $t \leqslant 5$, transient effects account for a large increase in amplitude, reaching a maximum $\left\|\boldsymbol{v}\left(t_{\max }\right)\right\| \approx 10^{3}$ for $t_{\max } \approx 2.5$; (ii) for $t>2.5$, the amplitude decays exponentially, i.e. $\mathrm{e}^{N t}$, with $N \approx-0.23$. In addition, the individual contributions of the pressure, the entropy and the velocity terms to the amplitude are represented.

The aim is then to understand the flow features responsible for both the transient growth and the asymptotic regime. The transient-growth mechanisms are analysed in terms of the instantaneous snapshots of the flow field and the evolution of the amplitude from $t=0$ to $t=5.5$ (figures 11-13). For each snapshot, the colour levels have been normalized by the $\infty$-norm of $\boldsymbol{v}(t)$. The flow dynamics in the asymptotic regime are assessed in terms of the entropy signal recorded at both surfaces of the aerofoil (figure 14). 


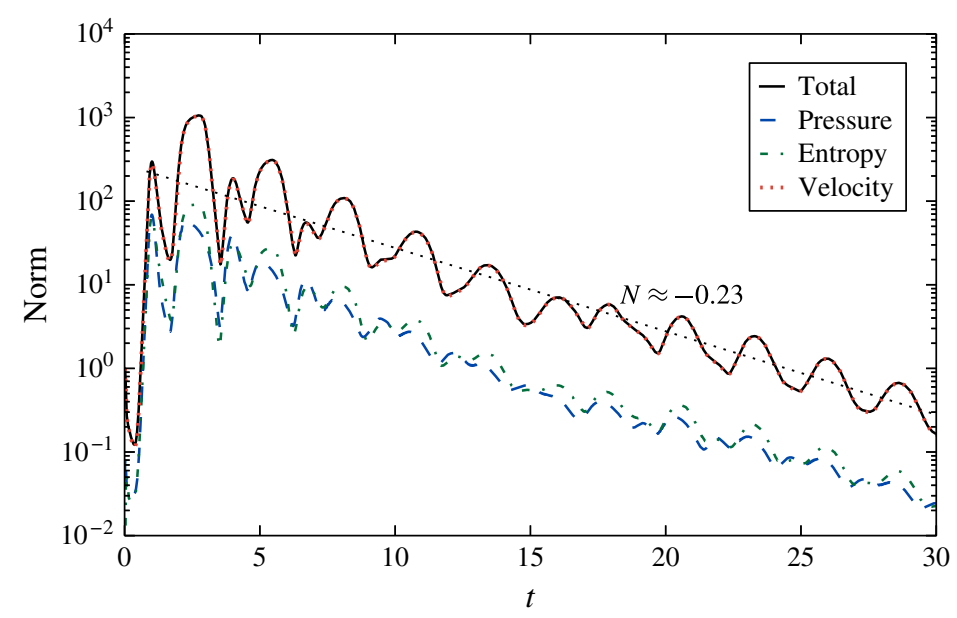

FIGURE 10. (Colour online) Impulse response of the linearized direct operator $\boldsymbol{A}$ showing the temporal evolution of the amplitude of the initial disturbance. Two regimes can be distinguished: for short times $t \leqslant 2.5$, the linearized operator exhibits important transient growth of the disturbances; for longer integration times $t>2.5$, the amplitude decays exponentially, verifying the stability of the linearized operator.

(a)

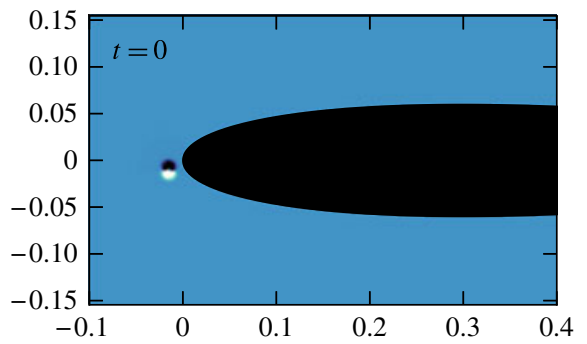

(c)

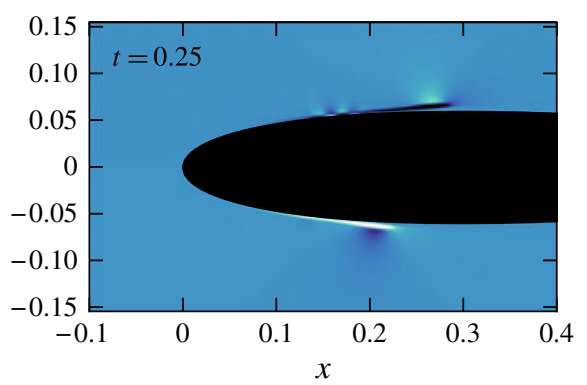

(b)

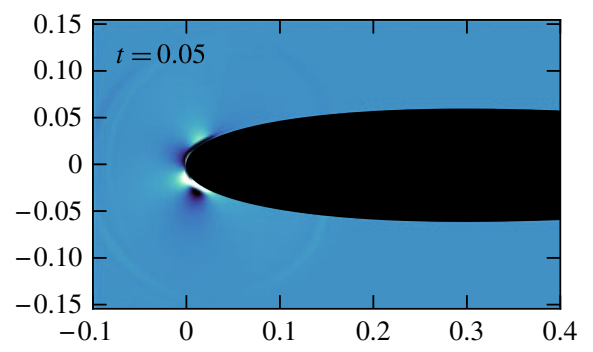

(d)

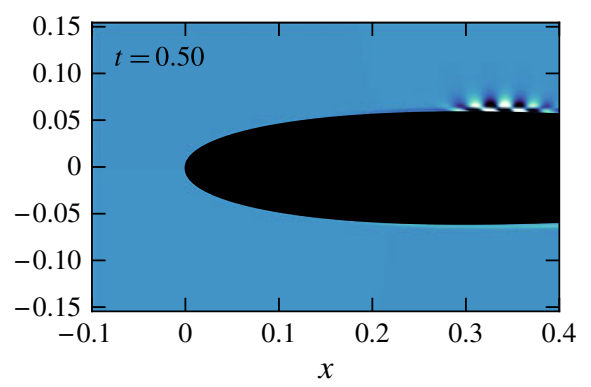

FIGURE 11. (Colour online) Initial growth of disturbances in the vicinity of the leading edge showing the dominant effect of the suction surface.

\subsection{Initial growth of disturbances: from the leading edge to the trailing edge}

Let us focus first on the transient-growth mechanisms that appear as the initial perturbation, displayed in figure 10 , is advected along the pressure and suction surfaces of the aerofoil. The decrease in amplitude at early stages $t<0.5$, figure 10 , can be interpreted in terms of the flow field evolution shown in figure $11(a-c)$. 
(a)

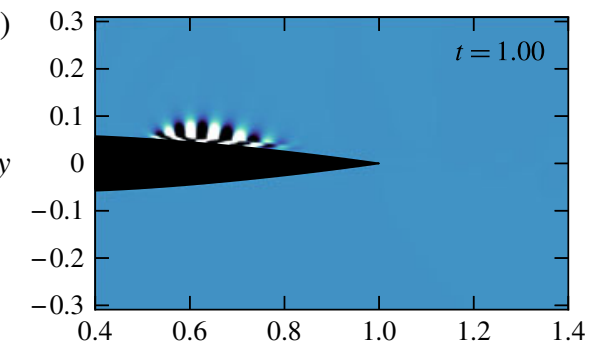

(c)

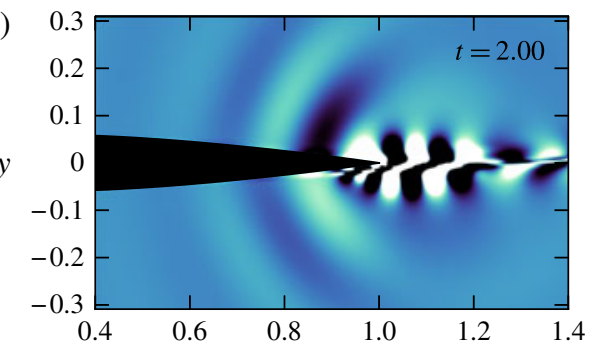

(e)

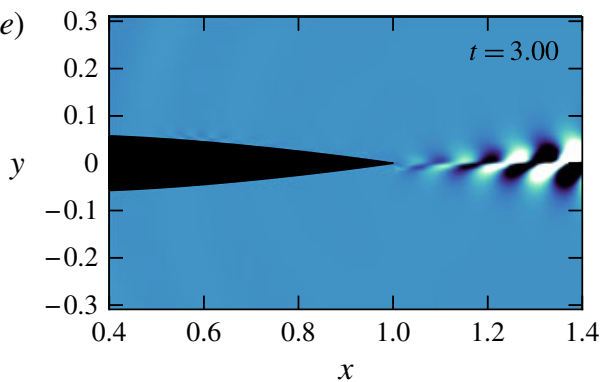

(b)

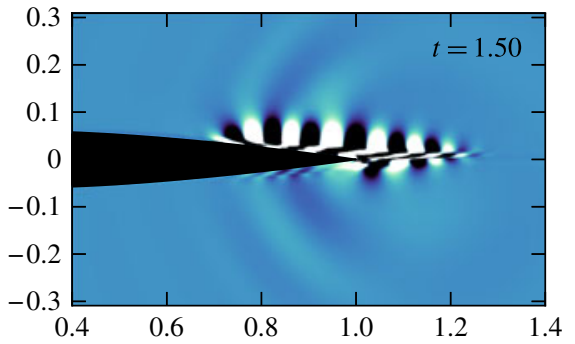

(d)

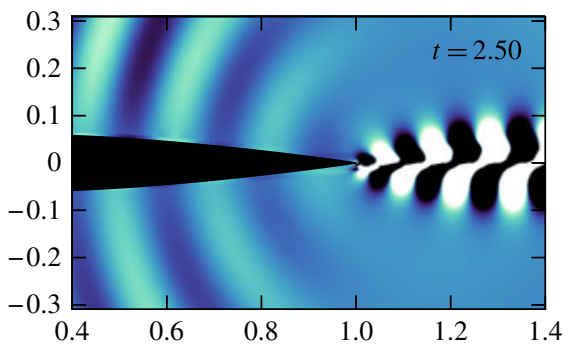

$(f)$

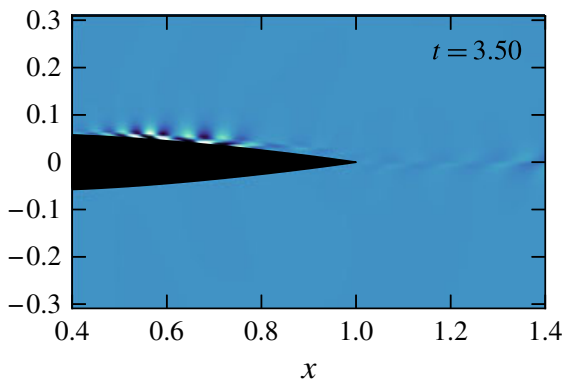

FIgURE 12. (Colour online) Analysis by impulse response: convective growth of instabilities on the pressure-surface and suction-surface boundary layers, followed by substantial acoustic radiation after the passing of disturbances at the trailing edge.

Owing to the transport of the disturbance by the base-flow velocity field, the perturbation separates into travelling perturbations along the suction and the pressure surfaces, and it experiences shear within the boundary layers; see figure $11(b, c)$. In more detail, and from a local point of view, the perturbation can be interpreted as a combination of acoustic, free-stream and boundary-layer modes. As the perturbation is advected, it evolves according to the local dispersion relation: each single mode is characterized by its respective phase speed, and grows or decays in space according not only to the local spatial growth rate, but also to the projection onto the modes of the immediate downstream profile. The advection speed of the emerging wavepackets is then given by the group velocity, since they consist of a superposition of local modes of the same branch.

At the edge of the boundary layer, the perturbation propagates at the free-stream velocity and at constant amplitude. However, a part of the perturbation within the boundary layer projects onto local boundary-layer modes, that may present spatial growth or decay. Close to the leading edge, the boundary layers are subjected to positive pressure gradients $(x<0.04$ for the suction surface and $x<0.24$ for the pressure surface) and, for the given flow regime, this indicates that the boundary-layer 

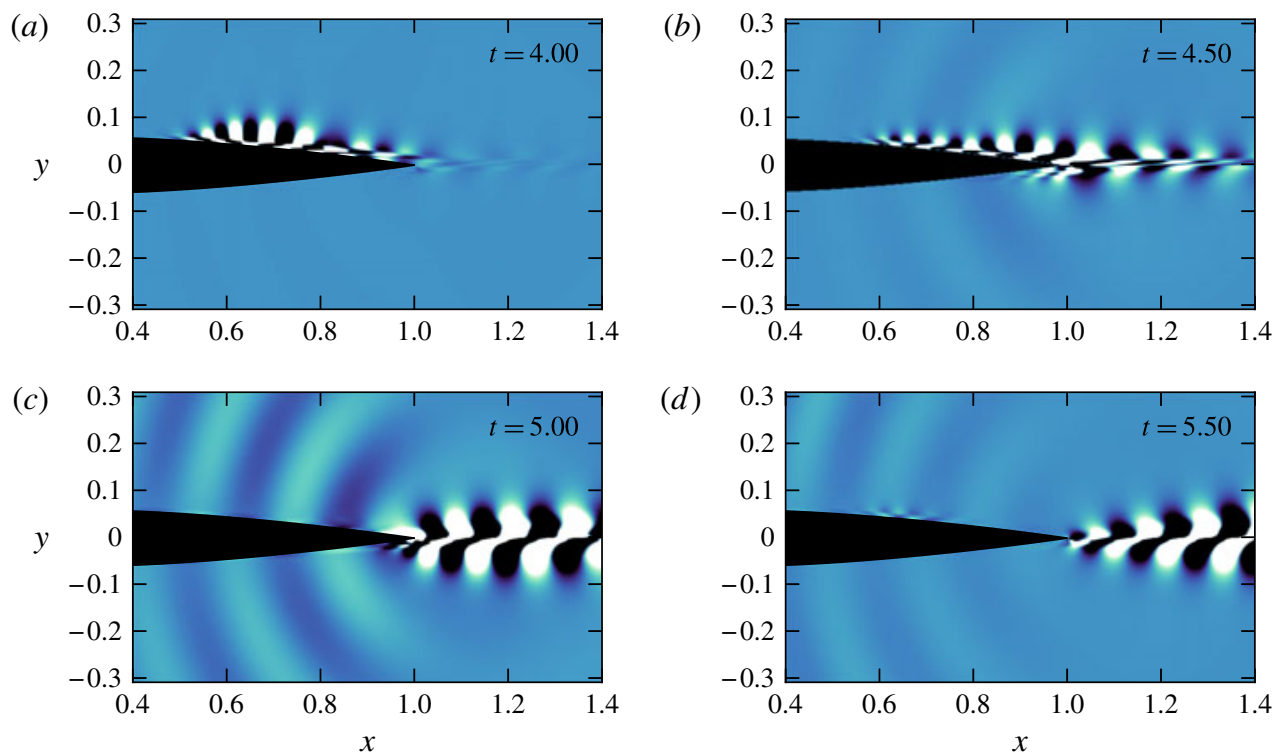

FIgURE 13. (Colour online) Analysis by impulse response: feedback loop showing instantaneous perturbation evolution, visualized by the streamwise velocity component.

(a)

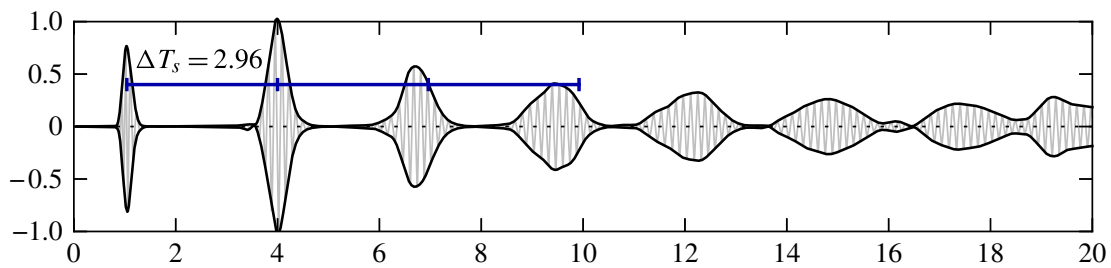

(b)

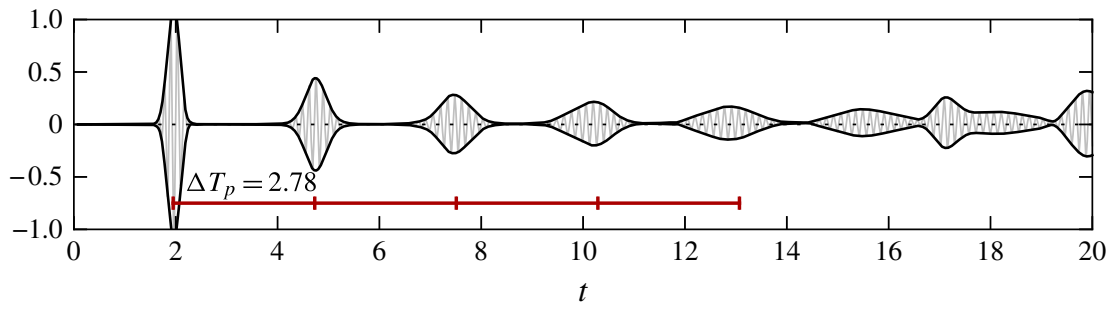

FIgURE 14. (Colour online) Analysis by impulse response: entropy signal at $(a)$ the suction-side probe $(x, y)=(0.6597,0.0474),(b)$ the pressure-side probe $(x, y)=$ $(0.9497,-0.0176)$, displaying the instantaneous signal in grey and the envelope in black.

modes are stable. The fraction of the perturbation that travels inside the boundary layer is then damped as it travels along convectively stable profiles, from where we can explain the initial decrease in amplitude of the perturbation.

In figure $11(d)$, the convective growth of a wavepacket on the suction surface can be attributed to inviscid-type instability mechanisms, since the local velocity profiles at these locations are inflectional (figure $8 a$ ). Contrarily, the pressure-surface boundary 
layer is expected to be convectively stable for $x<0.24$, explaining thus why the global linear dynamics for $0.5<t<1$ is predominantly localized on the suction surface.

The subsequent flow evolution is represented in figure 12. For $0.5<t<1$, the dominant mechanisms are related to Kelvin-Helmholtz instabilities within the shear layer of the suction-surface separation bubble. A local maximum in amplitude (figure 10) is reached at $t \approx 1$, and it coincides with the instant when the wavepacket approaches the reattachment point $x_{r, s} \approx 0.66$; see figure $12(a)$ and table 4 . For $1<t<1.6$, we observe a decrease in amplitude as the wavepacket propagates in the reattached flow. This behaviour can be related to a mismatch in the stability characteristics between the separation bubble and the reattached flow: the wavepacket does not project efficiently into convectively unstable waves in the reattached-flow region. After passing the trailing edge, the wavepacket produces scattering of acoustic waves in counter-phase between the upper and lower half-planes (figure 12b).

For $1.5<t<2.7$, the amplitude increases due to the growth of a wavepacket on the pressure-side separation bubble; see figure $12(b-d)$. Note that although perturbations are damped close to the leading edge, for $x>0.3$ the velocity profiles are inflectional (figure $8 b$ ) and therefore support spatial growth. It is reasonable to expect that the spatial growth rates of the attached - but inflectional - profiles are comparatively smaller than the spatial growth rates in the separation bubble where there is reverse flow. Indeed, by inspection of the flow field, one observes that most of the growth takes place in the shear layer of the separation bubble $0.7<x<1$.

A more intense acoustic radiation is observed as the pressure-surface wavepacket passes the trailing edge, compared to the suction surface wavepacket. Although in both cases the radiation has the same qualitative characteristics, it is found that: the frequency is slightly lower for the acoustic waves generated at the pressure surface than for the ones from the suction surface, and, more importantly, the acoustic field produced by the waves from the pressure surface is more intense than the one from the suction surface. For $2.7<t<3.5$, the wavepacket is located in the wake, where the dynamics is dominated by convection.

At this point, it is also important to state that no region of absolute instability, i.e. regions where the growth of wavepackets with zero group velocity takes place, exists in the flow. Further confirmation has been obtained by analysing the local velocity profiles: all velocity profiles exhibit negative temporal growth rates at the respective pinch points in the complex plane.

\subsection{Regeneration of wavepackets: feedback effects}

The flow evolution for $4<t<5.5$, depicted in figure 13, displays the same sequence of events that has been observed for $1<t<2.5$ : the wavepacket, issued from the boundary layer on the suction surface propagates downstream, emerges (figure 13a) and scatters acoustic waves as it passes by the trailing edge (figure 13b); a second wavepacket emerges on the pressure surface (figure 13b) and at its passage by the trailing edge, it radiates substantial acoustic energy into the far-field (figure 13c), generating in turn a wavepacket on the suction surface (figure 13d).

It is important to mention that for the first cycle, the instabilities were triggered by the initial perturbation at the leading edge. In contrast, for $3.5<t<5$, the triggering mechanism can be attributed to the receptivity of both boundary layers to free-stream disturbances originated downstream. This proposition is supported by the observation that the period between consecutive wavepackets is of the same order as the convective time over the aerofoil length. The above observation raises 
the question of whether the receptivity mechanism can mainly be attributed to the acoustic waves or to hydrodynamic disturbances induced by the wavepacket at the trailing edge.

On the one hand, the hydrodynamic features would excite the boundary layers upstream by a mechanism of forced receptivity due to a match in frequency and wavelength. On the other hand, the acoustic waves have the same frequency but a different wavelength than the hydrodynamic features and would thus require a mechanism of natural receptivity. Although the forced receptivity mechanisms are more efficient than the natural receptivity, it should be noted that the acoustic radiation decays algebraically with the distance from its source, whereas the hydrodynamic perturbations decay exponentially with the distance. Consequently, as far as the receptivity mechanism is concerned, the far-field acoustic radiation dominates over the far-field hydrodynamic disturbances (see, for instance, figure 13d).

\subsection{Cross-talk between the suction and pressure surface}

At this point, it is instructive to analyse the long-time behaviour in order to gain insight into the features of the feedback loop. To this end, we present in figure 14 the entropy signal, where the temporal decay has been factored out, recorded at the probes near the reattachment point of the suction-surface separation bubble $(x, y)=$ $(0.6597,0.0474)$, figure $14(a)$, and near the reattachment point of the pressure-surface separation bubble $(x, y)=(0.9497,-0.0176)$, figure $14(b)$.

For the transient regime observed before, we readily identify the period of wavepacket shedding on the suction surface as $\Delta T_{\mathrm{s}} \approx 2.96$, and that on the pressure surface as $\Delta T_{\mathrm{p}} \approx 2.78$. For longer times, $t>8$, there is an increase in complexity of the behaviour as the acoustic radiation, emitted by wavepackets from either surface, is prone to interact with the opposite boundary layer.

It is thus found that, for the present case, there exists a complex feedback loop involving hydrodynamic features on the pressure and suction surfaces of the aerofoil as well as in the near wake. The above features are related by feedback-loop effects due to the natural receptivity of the boundary layers to the upstream-propagating acoustic waves.

\section{Global stability analysis}

\subsection{Mathematical set-up and global mode calculation}

In this section we present a complementary approach to gain further insight into the description of the linearized dynamics: a more quantitative picture of the flow behaviour arises from the eigenvalue spectrum of the linearized operator $\boldsymbol{A}$. For this we assume a modal decomposition into elementary solutions of the form $v_{k} \exp \left(-\mathrm{i} \omega_{k} t\right)$, where the direct global mode $\boldsymbol{v}_{k}$ and the global frequency $\omega_{k}$ are given by the solution of the eigenvalue problem

$$
\boldsymbol{A} \boldsymbol{v}_{k}=-\mathrm{i} \omega_{k} \boldsymbol{v}_{k} \text {. }
$$

The above decomposition allows us to determine and categorize the physical mechanisms that are present in the flow. At early times, all direct modes (regardless of their growth rate) may be important for the dynamics of the flow. On a long time scale, the dynamics is mainly governed by the least-stable global modes. In this section, we focus first on the features of the least-stable direct global modes, which may play an important role in the observed feedback loop and, more importantly, may be linked to the structure of the acoustic spectrum in the nonlinear simulations as well as in experimental investigations. 


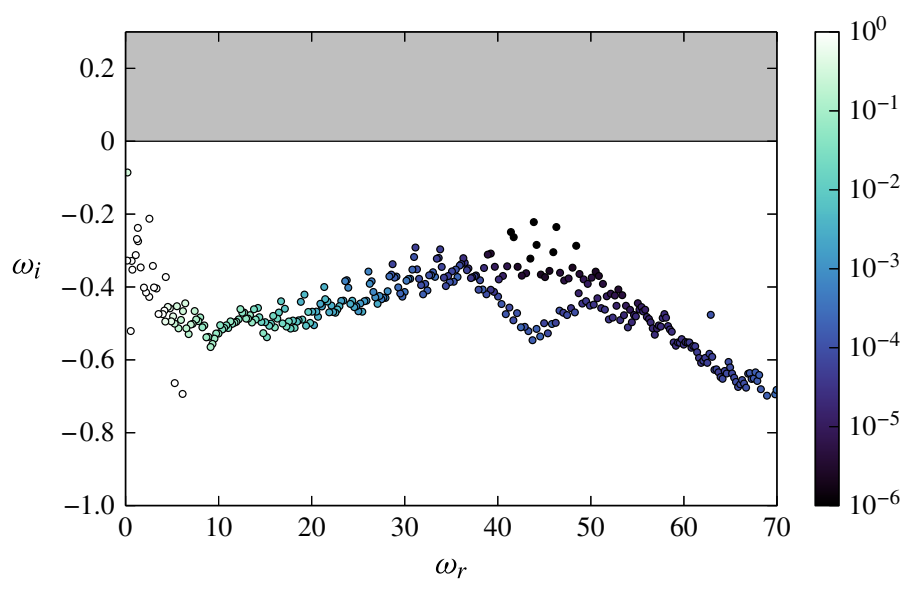

FIGURE 15. (Colour online) Global spectrum of the tonal noise problem in the complex frequency plane. The greyscale indicate the relative residual with respect to the operator $\boldsymbol{A}$.

\subsubsection{Numerical details on the global mode calculations}

Prior to launching into the global modes analysis, we summarize here the parameters of the numerical calculation that permit us to obtain the global spectrum. In order to compute the least-stable global modes, we apply the Krylov-Schur technique (Stewart 2002), as implemented in the eigensolver SLEPc (Hernández, Román \& Vidal 2005), to the operator that represents the temporal advancement over time $\Delta t$ for the direct operator $\mathrm{e}^{\Delta t A}$. This approach is usually known as the time-stepper method (Edwards et al. 1994).

In the present calculations, the size of Krylov subspaces is 2048, and the time step between consecutive snapshots is taken as $\Delta t=0.015$. The results from the nonlinear simulations (table 3 ) hint at $\omega \approx 44$ as the most probable location for the global modes of interest. In order to improve the convergence of the eigenvalues at this location in the complex plane, we have enabled the use of the harmonic extraction technique in the eigensolver with the shift parameter $\sigma$ as $\mathrm{e}^{\mathrm{i} \Delta t \omega}$. It is important to mention that the estimate of the relative error with respect to the propagator is commonly more optimistic than the actual error. For this reason, the degree of convergence is measured according to the relative residual $\varepsilon_{k}=\left\|\boldsymbol{A} \boldsymbol{v}_{k}+\mathrm{i} \omega_{k} \boldsymbol{v}_{k}\right\|_{2} /\left\|\omega_{k} \boldsymbol{v}_{k}\right\|_{2}$.

\subsection{Features of the global spectrum}

The computed global spectrum is depicted in figure 15, showing the location of global modes in the complex plane $\omega=\omega_{r}+\mathrm{i} \omega_{i}$ according to the angular frequency $\omega_{r}$ and the temporal growth rate $\omega_{i}$. Since the operator $\boldsymbol{A}$ is real, the spectrum is symmetric with respect to the vertical axis, and it thus suffices to focus on the features of the global modes in the right half-plane.

A first inspection of figure 15 confirms that the operator $\boldsymbol{A}$ is stable, since all the eigenvalues fall into the lower half of the complex plane $\omega_{i}<0$. No isolated branches of global modes can be observed in the spectrum. For increasing frequency, the growth rate associated with the least-damped modes shows two maxima: at $\omega \approx$ $43.87-0.22 \mathrm{i}$ and close to the origin $\omega \approx 0$. These maxima correspond, within a reasonable approximation, to the main frequencies observed in the acoustic tones from the nonlinear simulations $\omega \approx 41.84$ (see table 3 ) and low-frequency components. 

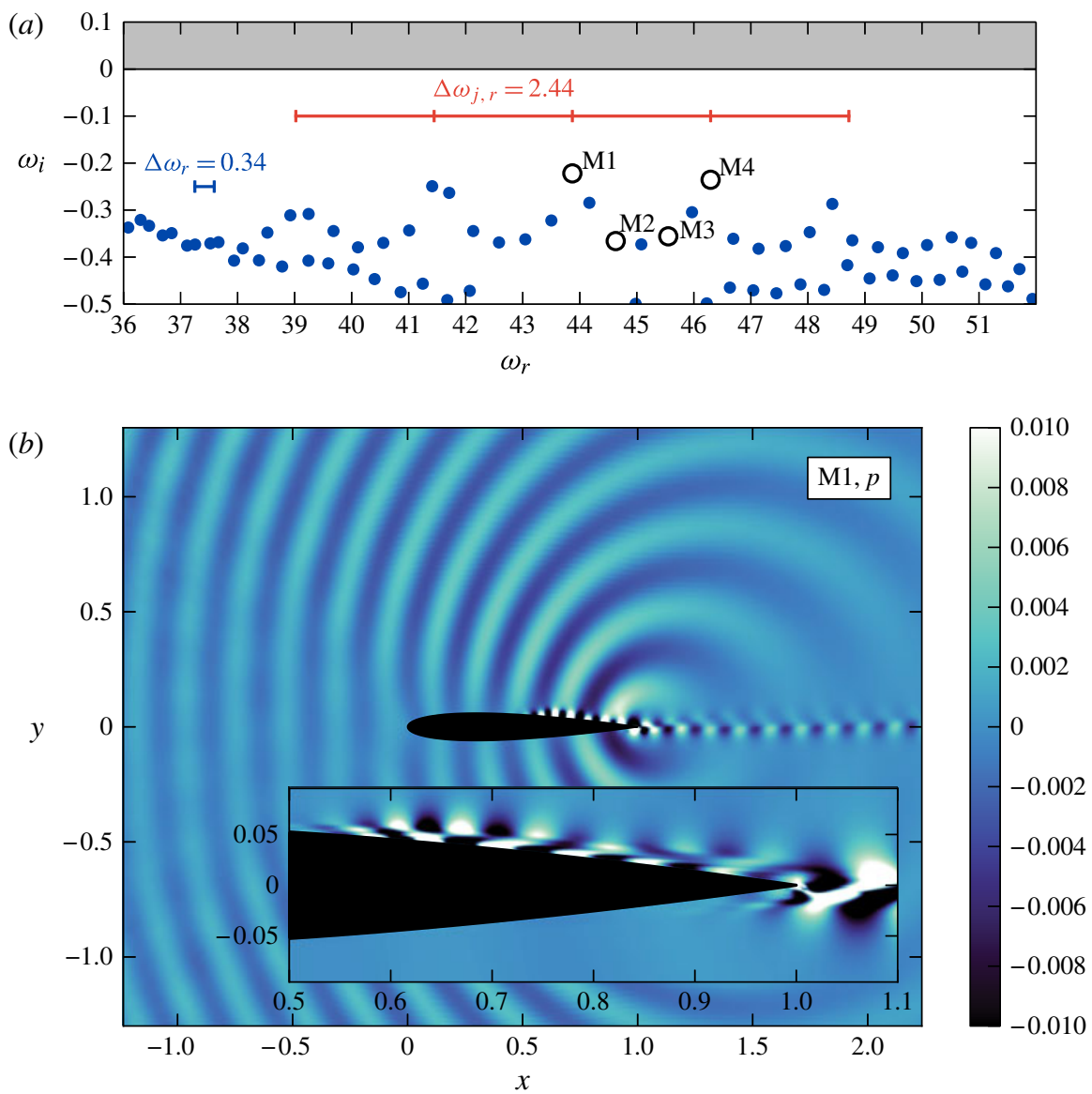

FIGURE 16. (Colour online) (a) Detailed view of the global spectrum showing the least-stable (or leading) global modes. The modes M1-M4 are considered, and the corresponding eigenvalues are $\omega_{\mathrm{M} 1} \approx 43.87-0.22 \mathrm{i}, \omega_{\mathrm{M} 2} \approx 44.63-0.37 \mathrm{i}, \omega_{\mathrm{M} 3} \approx 45.55-$ $0.36 \mathrm{i}$ and $\omega_{\mathrm{M} 4} \approx 46.29-0.24 \mathrm{i}$. The relative residuals for the modes shown fall below $10^{-5}$. (b) Spatial structure of the global mode labelled M1, displaying the real part of the associated near-field pressure levels, and the real part of the streamwise velocity levels in the vicinity of the aerofoil surface (inset). The mode presented here has been normalized by the maximum value of the velocity field in the near wake $1<x<1.2$.

\subsection{Leading modes: the coupled dynamics of the separation bubbles}

Figure 16(a) provides a detailed view of the spectrum for the subdomain comprising the leading global modes. It reveals that the leading global modes consist of multiple local maxima in growth rate centred around the maximum growth-rate peak at $\omega_{r} \approx 43.87$ and $\omega_{i}=-0.22$. The frequency difference between consecutive maxima is nearly constant and equal to $\Delta \omega_{r} \approx 2.44$, and the temporal growth for the local maxima is approximately $\omega_{i} \approx-0.22$. Between these peaks, the frequency difference of neighbouring eigenvalues is $\Delta \omega_{r}=0.34$, and their minimum growth rate is approximately $\omega_{i}=-0.35$.

We next consider the mode labelled M1 in the spectrum (see figure 16a) corresponding to the local maximum in growth rate, represented in figure $16(b)$ 

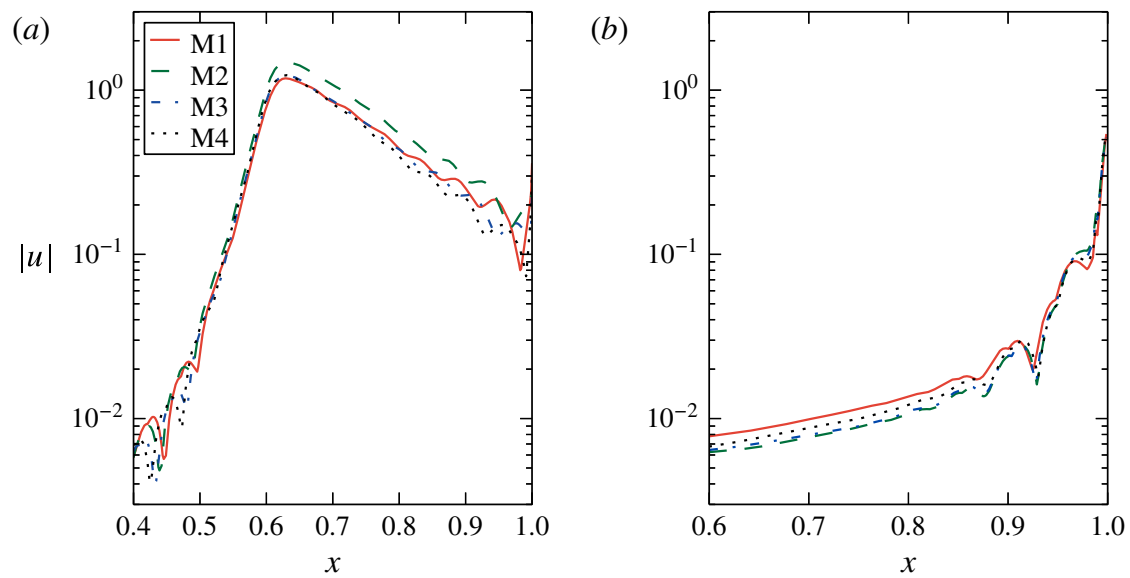

FIGURE 17. (Colour online) Evolution of the streamwise velocity peak along the aerofoil chord $(a)$ on the suction surface and $(b)$ on the pressure surface of the aerofoil for the modes labelled M1-M4 in figure 16.

by the real part of the streamwise velocity component for the downstream half of the aerofoil (inset) and the real part of the associated pressure in the near field of the aerofoil.

In order to facilitate a comparison, the leading global mode has been normalized by the maximum value of the velocity in the near wake $1<x<1.2$, which for the displayed modes occurs at approximately $x=1.15$. From the velocity fields at the aerofoil, we conclude that the modes represent shear-layer instabilities with predominant spatial support downstream of the reattachment point of the suction-surface separation bubble and in the wake. For increasing frequency the characteristic wavenumber of the oscillations increases. Under the chosen scaling, the spatial growth becomes noticeable on the suction surface for $x>0.5$ and on the pressure surface for $x>0.95$. These locations fall within the respective regions given by the separated shear layer and the reattachment point of the separation bubble. Therefore, we can deduce that the family of global modes in the range $40<\omega<50$ represents the coupled global dynamics of both separation bubbles.

In the pressure field we observe the acoustic footprint of the boundary-layer instabilities. In the near field, the mode displays substantial levels of acoustic radiation into the free stream with higher wavelengths. The radiation can be attributed to a dipolar acoustic source contained in the global mode at the trailing edge, whose acoustic pattern corresponds to cylindrical waves in counter-phase between the upper and lower half-planes. Furthermore, the acoustic waves show a preferred upstream directionality.

The chosen global modes M1-M4 exhibit over a range of frequencies nearly identical features in both the hydrodynamic instabilities and the associated acoustic radiation. We then focus on the evolution of the maximum peak of the streamwise velocity (figure 17) and of the pressure peak (figure 18) on both surfaces of the aerofoil. The evolution of the streamwise velocity peak on the suction surface (figure 17) displays the exponential growth of the instability waves at $0.53<x<0.62$. This range coincides with the separated shear layer at the edge of reattachment. As already hinted by the impulse response analysis, further downstream the mode decays exponentially in space until it reaches the trailing edge. Further upstream, the velocity 
(a)

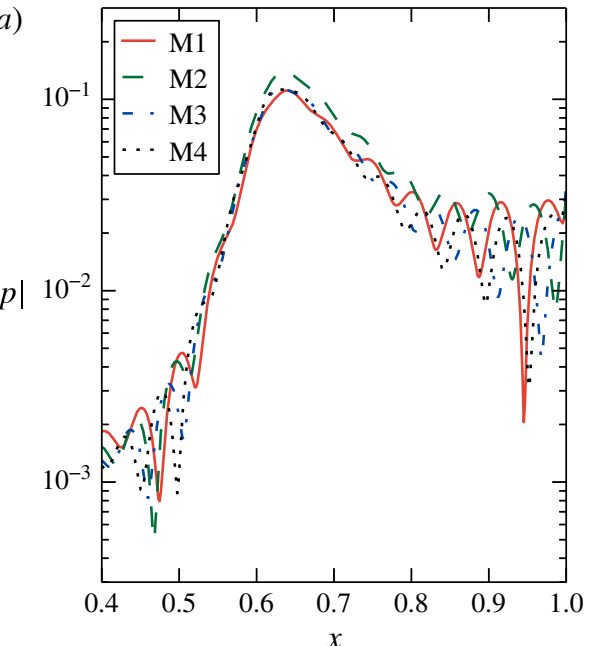

(b)

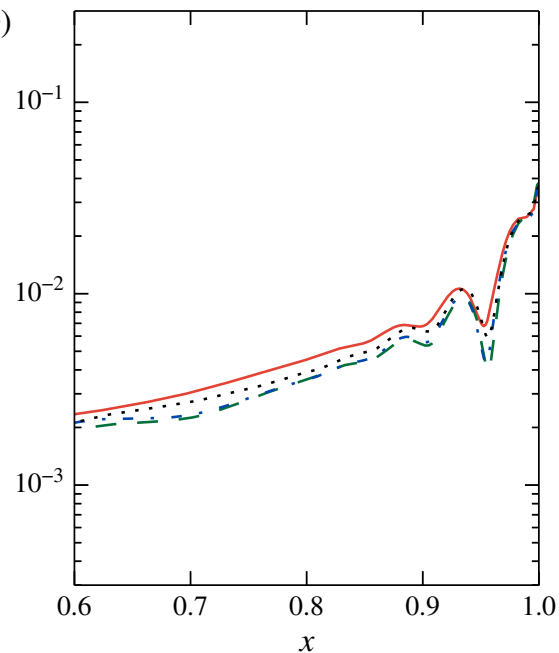

FIGURE 18. (Colour online) Evolution of the pressure peak along the aerofoil chord $(a)$ on the suction surface and $(b)$ on the pressure surface of the aerofoil for the modes labelled M1-M4 in figure 16.

values are approximately constant (in logarithmic scale) and they correspond to the levels of velocity fluctuations outside the boundary layer. On the pressure surface, the exponential growth of streamwise velocity takes place near the trailing edge $0.9<x<1$, where the base flow exhibits a separated shear layer at the location of the reattachment. Similarly to what has been observed for the suction side, upstream values of the velocity are nearly constant (in logarithmic scale) and are related to velocity fluctuations outside the boundary layer.

The evolution of the pressure fluctuations on the suction and pressure surfaces reveals the appearance of standing waves on the suction surface and, to a lesser extent, on the pressure surface. On the suction surface, the nodes of the standing wave are located at $x \approx 0.5$ and are uniformly distributed for $0.8<x<1$. Note that their location varies significantly for the different modes. On the pressure surface, however, the node is located at $x \approx 0.95$. Detailed inspection shows that the modes with the higher growth rates (M1 and M4) are correlated with the location of the suction-surface node at $x \approx 0.95$, which corresponds to the location of the node on the pressure surface.

It is important to point out that the identified region of dominant exponential growth does not necessarily imply that all growth takes place at this location: upstream, the amplitude of the hydrodynamic perturbations is far smaller than the induced level of fluctuations associated with the acoustic waves. Since the acoustic waves and the hydrodynamic perturbations occur at the same frequency, we are unable to determine precisely the exact location of the origin of exponential growth.

At this point, it is convenient to compare the occurrence of the local maxima in the global spectrum with the location of the equally spaced tones in the acoustic spectrum; see table 5. For frequencies where this comparison can be performed, the agreement between the acoustic tones and the spacing in the global spectrum is excellent: the maximum relative error is $1.1 \%$. These observations suggest that the tonal noise phenomenon and the occurrence of multiple equally spaced peaks in the acoustic spectrum have their physical origin reflected in the characteristics of the 


\begin{tabular}{lccccc} 
& \multicolumn{6}{c}{ Frequency peaks } \\
\cline { 2 - 6 } Case G1 & $\omega_{-2}$ & $\omega_{-1}$ & $\omega_{0}$ & $\omega_{1}$ & $\omega_{2}$ \\
Linear: global modes & - & 39.24 & 41.38 & $\mathbf{4 3 . 8 7}$ & 46.30 \\
Nonlinear: Fourier & 37.02 & 39.47 & $\mathbf{4 1 . 9 3}$ & 44.38 & - \\
Relative difference & - & $0.5 \%$ & $1.1 \%$ & $1 \%$ & -
\end{tabular}

TABLE 5. Comparison between the tones in the acoustic spectrum for the nonlinear simulation (see table 3, case G1) and the frequency of the global modes with local maxima of temporal growth rate in the global spectrum. The peak with maximum growth rate and the loudest tone in the nonlinear simulation are indicated in bold.

global spectrum, and, more importantly, the principal mechanism can be ascribed to the linearized dynamics of the flow.

We observed in the impulse response analysis (in $\S 3$ ) that, triggered by the passage of perturbations at the trailing edge of the aerofoil, an aeroacoustic feedback loop establishes itself and subsequently drives the disturbance evolution. The features of this feedback loop were then described phenomenologically in terms of the dynamics of hydrodynamic and acoustic wavepackets, and their origin was linked to local properties of the base flow. Even though for short times we observed distinct wavepackets, for longer times, we could no longer identify individual wavepackets but rather high-frequency components modulated by low-frequency oscillations with a period of $T_{f} \approx 2.8$. With the direct global modes extracted, we are now in a position to provide a complementary description of the feedback loop in terms of the dynamics of the least-stable modes: for sufficiently long time, the temporal evolution is mainly given by a superposition of the least-stable global modes, since the remaining modes have comparatively smaller growth rates. As was discussed before, the global modes display the most relevant physical features that are also observed in the nonlinear simulations, matching in spatial structure as well as in frequency. The question remains, then, of how the global modes can account for the above feedback loop.

To answer this question, we consider the superposition of two plane waves with similar spatio-temporal characteristics: their respective frequencies are taken as $\bar{\omega}+\Delta \omega$ and $\bar{\omega}-\Delta \omega$, and the wavenumbers are $\bar{\alpha}+\Delta \alpha$ and $\bar{\alpha}-\Delta \alpha$. Hence their superposition is given by

$$
\begin{aligned}
A(x, t) & =\exp (\mathrm{i}[(\bar{\alpha}+\Delta \alpha) x-(\bar{\omega}+\Delta \omega) t])+\exp (\mathrm{i}[(\bar{\alpha}-\Delta \alpha) x-(\bar{\omega}-\Delta \omega) t]) \\
& =2 \cos \left(\Delta \alpha\left(x-c_{g} t\right)\right) \exp \left(\mathrm{i} \bar{\alpha}\left(x-c_{p} t\right)\right),
\end{aligned}
$$

with the phase velocity $c_{p}=\bar{\omega} / \bar{\alpha}$ and the group velocity $c_{g}=\Delta \omega / \Delta \alpha$. From this expression, it is readily observed that for $\Delta \omega \ll \bar{\omega}$ and for $\Delta \alpha \ll \bar{\alpha}$ the evolution can be interpreted as oscillations at the average frequency $\bar{\omega}$ and wavenumber $\bar{\alpha}$, modulated by a low-frequency component at half the frequency difference $\Delta \omega$ and half the wavenumber difference $\Delta \alpha$. Moreover, the velocity of the low-frequency component is given by the group velocity $c_{g}$, which is different from the phase velocity of the high-frequency component $c_{p}$. In other words, the waves display beating, which arises from their linear superposition and is linked to their spatio-temporal resemblance.

In an analogous manner, global modes with similar spatial structures and nearly matched frequencies can also exhibit beating. The evolution of the flow can be 
described by a superposition of global modes $\boldsymbol{v}(t)=\sum_{i} a_{i} \boldsymbol{v}_{i} \exp \left(-\mathrm{i} \omega_{i} t\right)$. For each pair of modes $\boldsymbol{v}_{k}$ and $\boldsymbol{v}_{l}$, we can write their superposition as

$$
a_{k} \boldsymbol{v}_{k} \mathrm{e}^{-\mathrm{i} \omega_{k} t}+a_{l} \boldsymbol{v}_{l} \mathrm{e}^{-\mathrm{i} \omega_{l} t}=2[\cos (\Delta \omega t) \overline{\boldsymbol{v}}-\mathrm{i} \sin (\Delta \omega t) \Delta \boldsymbol{v}] \mathrm{e}^{-\mathrm{i} \bar{\omega} t},
$$

where $\bar{\omega}=\left(\omega_{l}+\omega_{k}\right) / 2, \Delta \omega=\left(\omega_{l}-\omega_{k}\right) / 2, \overline{\boldsymbol{v}}=\left(a_{l} \boldsymbol{v}_{l}+a_{k} \boldsymbol{v}_{k}\right) / 2$, and $\Delta \boldsymbol{v}=\left(a_{l} \boldsymbol{v}_{l}-a_{k} \boldsymbol{v}_{k}\right) / 2$. The above expression shows that the same decomposition in terms of a high-frequency carrier wave and a low-frequency modulation can be performed. In the context of fluid dynamics, this beating between global modes has been observed in several fluid cases; see for instance Schmid \& Henningson (2002) for the case of a falling liquid curtain and Ehrenstein \& Gallaire (2008) and Cherubini et al. (2010) for the beating of separation bubbles.

For the present case, we can estimate the typical period of the feedback loops from half the difference between the peaks at both extremes of the leading modes; we get $\Delta \omega=2.44$. This estimate is in good agreement with the angular frequency associated with the period of the feedback loops that was observed in the impulse response: $\omega_{p}=2 \pi / \Delta T_{p} \approx 2.26$. The difference in frequency can be attributed to the fact that the full interaction involves multiple modes with similar frequencies, which complicates the mechanism. The propagation velocity of the wavepackets is given by the group velocity $c_{g}=\Delta \omega / \Delta \alpha$, where $\Delta \alpha$ is obtained from the spatial structure of the global modes involved in the feedback loop.

From a global perspective, it can be concluded that the feedback loop that appears in the impulse response (and that has also been observed in the nonlinear simulation) arises from the interaction of the leading global modes with similar frequencies. Their modal interaction describes the growth of wavepackets on the suction and pressure surfaces and includes the radiation of acoustic waves. We emphasize that the feedbackloop phenomenon cannot be attributed to any individual global mode.

\subsection{High-frequency modes: suction-surface shear-layer instabilities}

Our attention is now directed towards the features of the remaining modes of the spectrum. We present in figure 19 a closer view of the global spectrum for the range of high frequencies $\omega_{r}>50$.

This part of the global spectrum contains modes with increasingly lower temporal growth rates, representing short-time-scale dynamics. Four representative modes, labelled $\mathrm{H} 1-\mathrm{H} 4$ in figure 19, are depicted in figure $19(b-e)$, visualized by the real part of the streamwise velocity. The high-frequency direct modes have been scaled by the maximum value of the streamwise velocity on the suction surface, which for the displayed modes occurs at approximately $x=0.60$.

The direct mode $\mathrm{H} 1$, depicted in figure $19(b)$, displays features similar to the coupled dynamics between the suction-surface and pressure-surface separation bubbles that was observed for the leading modes in the frequency range $30<\omega_{r}<50$. Although acoustic radiation is observed, it is comparatively smaller than that of the leading modes. Owing to the higher frequency, the spatial scales are noticeably shorter than those of the leading modes M1-M4. For even higher frequencies (figure 19c) we observe a qualitative change from mode $\mathrm{H} 1$ to mode $\mathrm{H} 4$ : the direct global mode reduces the spatial support in the near wake of the aerofoil, and on the suction surface, the front of the global mode moves upstream into the shear-layer region. In the reattached flow region, the mode describes the advection by the free stream, lacking significant interaction with the boundary layer. Furthermore, the acoustic radiation disappears for higher-frequency modes $(\mathrm{H} 2-\mathrm{H} 4)$. The transition from $\mathrm{H} 1$ to 

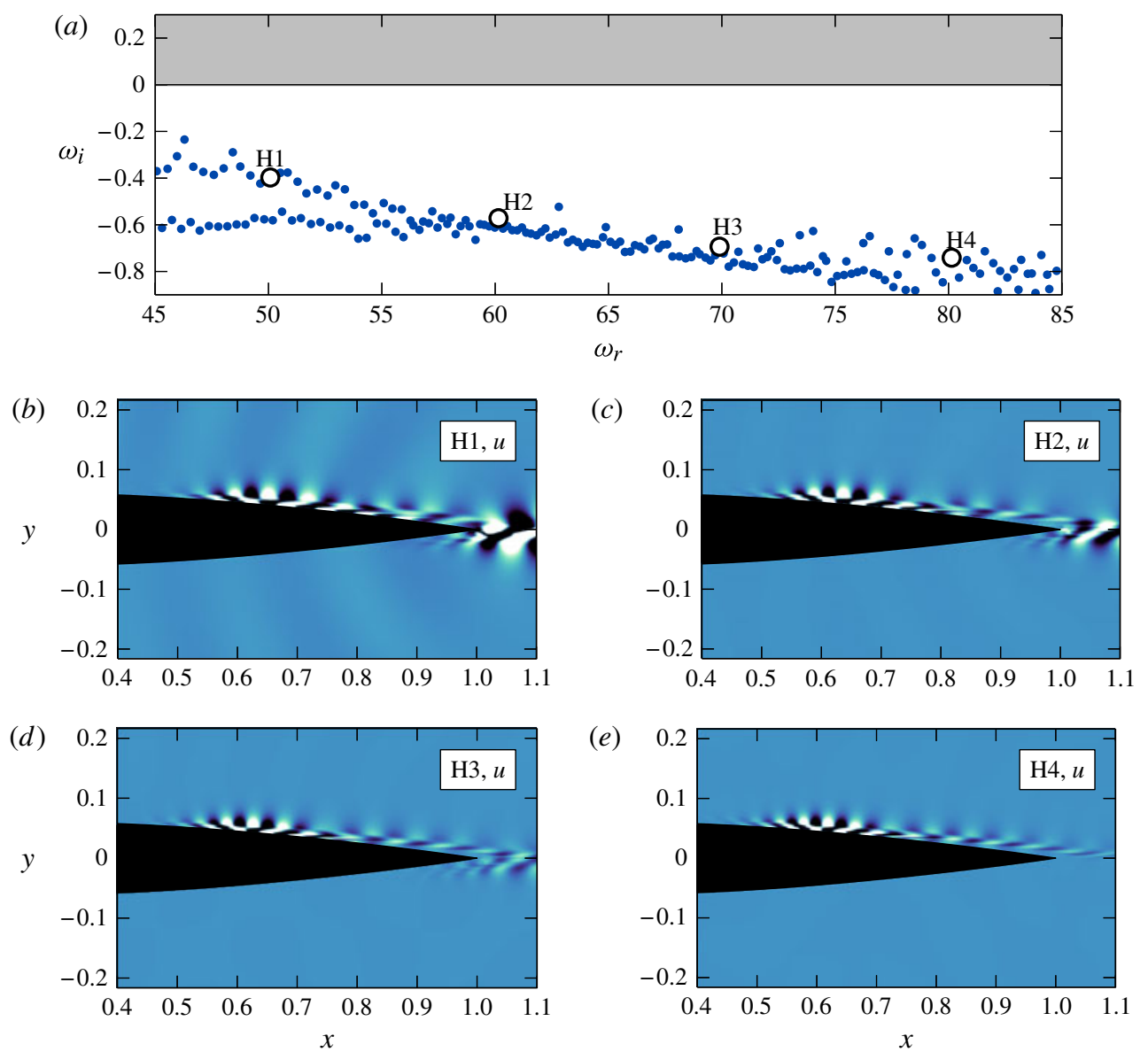

FIGURE 19. (Colour online) Part of the (a) global spectrum displaying high-frequencies $\omega_{r}>50$ and representative direct global modes, visualized by the real part of the streamwise velocity $u$. The labels $\mathrm{H} 1-\mathrm{H} 4$ correspond to the eigenfrequencies of the direct global modes shown in $(b) \omega_{\mathrm{H} 1} \approx 50.08-0.40 \mathrm{i},(c) \omega_{\mathrm{H} 2} \approx 60.15-0.57 \mathrm{i},(d) \omega_{\mathrm{H} 3} \approx$ $69.89-0.69 \mathrm{i}$ and $(e) \omega_{\mathrm{H} 4} \approx 80.14-0.74 \mathrm{i}$. The residual for the modes shown fall below $1 \times 10^{-3}$.

H4 can be explained by returning to the characteristic scales of the different separated shear layers. In order to comply with the prevalent dispersion relations, the spatial support of the mode shows shorter wavelengths for increasing frequencies. Since the thickness of the separated shear layer is smaller on the suction side than on the pressure side, for short wavelengths (or higher frequencies) the suction-surface separation bubble supports instabilities whereas the growth on the pressure surface is less pronounced.

\subsection{Low-frequency modes: flapping of the separation bubbles and reattached flow dynamics}

The global spectrum for $\omega<35$ is displayed in figure 20. As before, we have chosen four representative modes $\mathrm{L} 1-\mathrm{L} 4$ in order to investigate the features of the global 

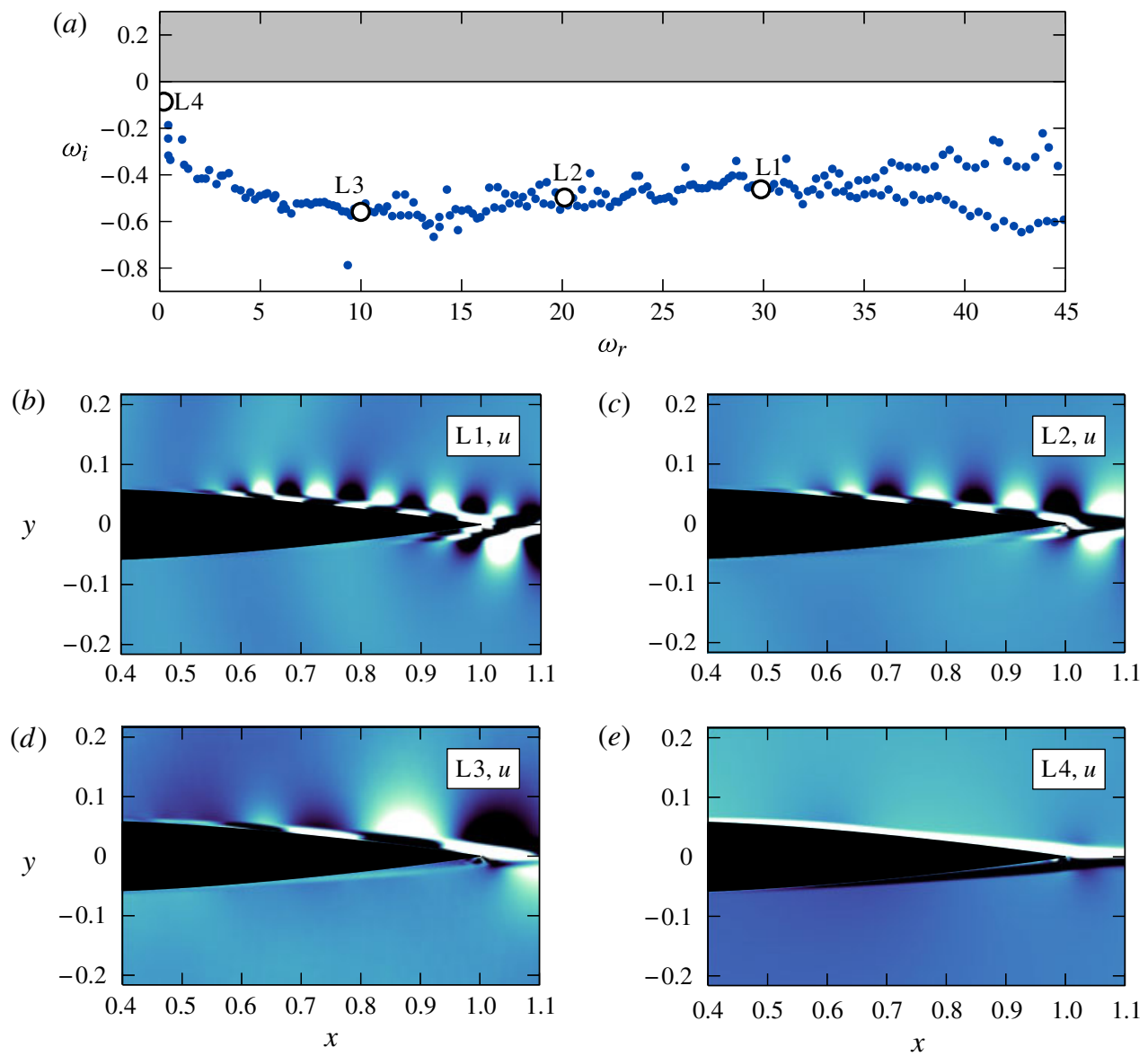

FIgURE 20. (Colour online) Part of the (a) global spectrum displaying low frequencies $\omega_{r}<35$. Representative global modes, labelled L1-L4 and visualized by the real part of the streamwise velocity $u$, are shown in $(b) \omega_{\mathrm{L} 1}=29.88-0.46 \mathrm{i}$, (c) $\omega_{\mathrm{L} 2}=20.12-0.50 \mathrm{i}$, (d) $\omega_{\mathrm{L} 3}=9.99-0.56 \mathrm{i}$, and $(e) \omega_{\mathrm{L} 4}=0.20-0.09 \mathrm{i}$.

spectrum for decreasing frequency. The normalization criterion is identical to the one described for the high-frequency modes.

The global mode L1 shown in figure 20(b) corresponds to the lower bound in frequency (and wavenumber) of the modes that represent the coupled dynamics of the separation bubble on both surfaces. Contrary to the previous case, for decreasing frequencies we expect an increase in the wavelength of the observed instabilities; the corresponding global modes should display structures in the shear layers that exceed the scale of the separation bubbles. Modes L2 and L3 confirm that for lower frequencies (figure $20 c, d$ ) the global modes undergo a qualitative change: the direct global modes localize downstream of the separation bubble, in the region where the flow is reattached. For the lowest-frequency mode L4 the wavelength is comparable with the extent of the separation bubbles; this mode represents synchronized low-frequency oscillations, in opposite phase, between both separation bubbles (figure 20e). 

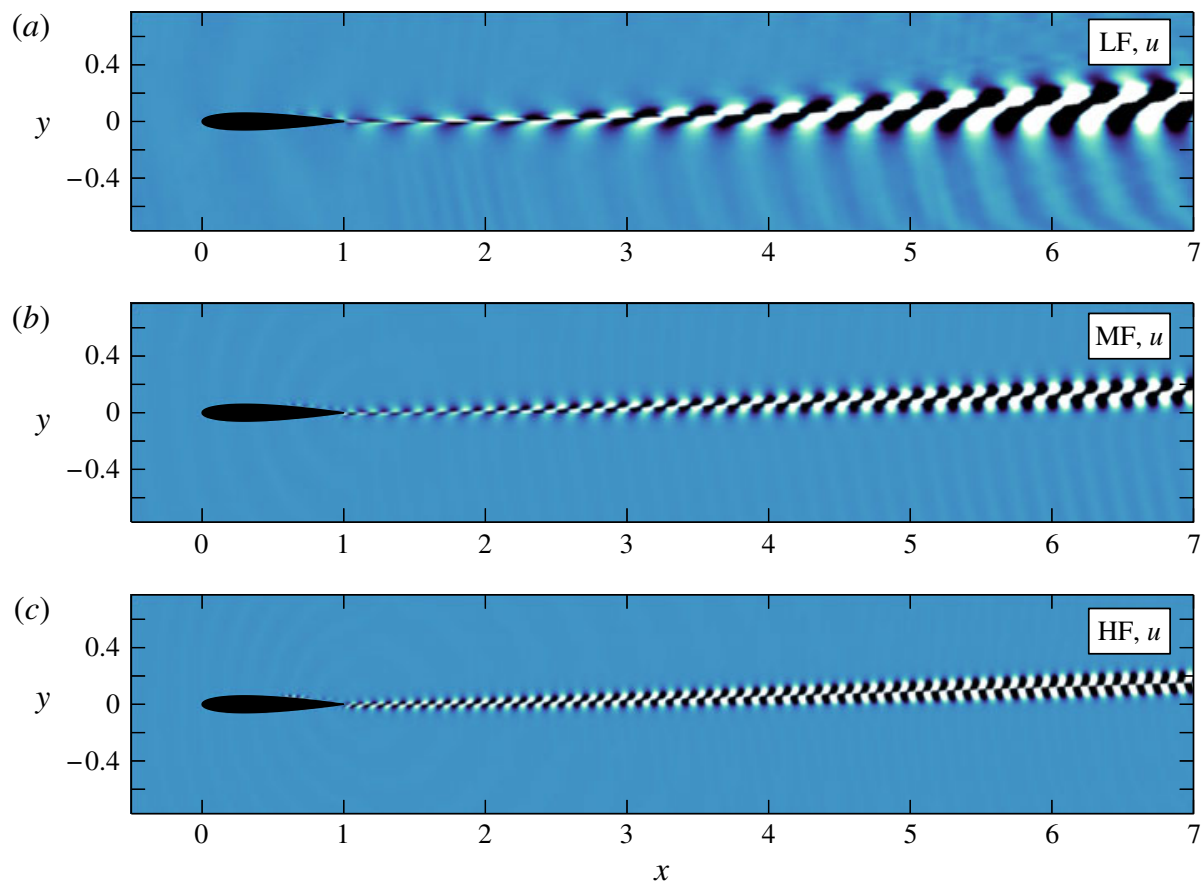

FIGURE 21. (Colour online) Downstream advection effects for the computed modes showing the real part of the streamwise velocity of $(a)$ low-frequency modes $\omega_{\mathrm{LF}}=$ $15.33-0.55 \mathrm{i},(b)$ leading modes $\omega_{\mathrm{MF}}=35.02-0.48 \mathrm{i}$ and $(c)$ high-frequency modes $\omega_{\mathrm{HF}}=55.08-0.51 \mathrm{i}$.

\subsection{Effect of convection on the computed global modes}

In the description of the modal dynamics, we have so far focused on the dynamics in the vicinity of the aerofoil surface and the near wake. However, there also exist global modes that capture the advection of perturbations in the wake as well as the propagation of acoustic waves in the far field.

In figure $21(a-c)$ we present the real part of the streamwise velocity for a representative set modes from each of the families of global modes that have been identified above. The global modes have been chosen such that the temporal growth rate is approximately equal to $\omega_{i} \approx-0.5$. A common characteristic for the selected modes is the exponential growth in the wake.

The global modes presented above describe the dynamics of instability waves in the computational domain. These waves reach the trailing edge, after which they are subjected to advection by the base flow (note that the base flow does not exhibit significant velocity deficit in the wake). This advective part is characterized by a (non-dimensionalized) dispersion relation of the form $\omega=\alpha$. As a consequence, the spatial structure of the global mode in the wake necessarily displays spatial, exponential growth. In figure $22(a)$ we display the magnitude of the streamwise velocity in the wake using a logarithmic scale. Since $\omega_{i} \approx-0.5$, the spatial growth rate in the wake can be approximated as $-\alpha_{i} \approx 0.5$, as confirmed in the figure. A similar phenomenon is observed in figure $22(b)$ for the evolution of the pressure amplitude in the far field along a vertical line through the trailing edge for the modes M1-M4; in this case the radial decay of acoustic waves has to be taken into account. 
(a)

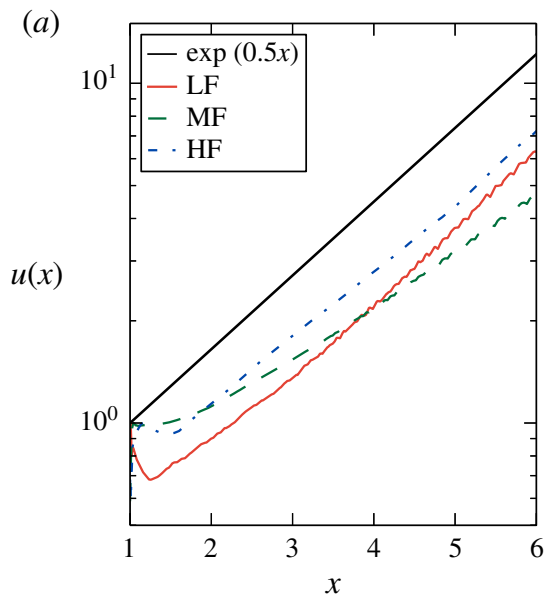

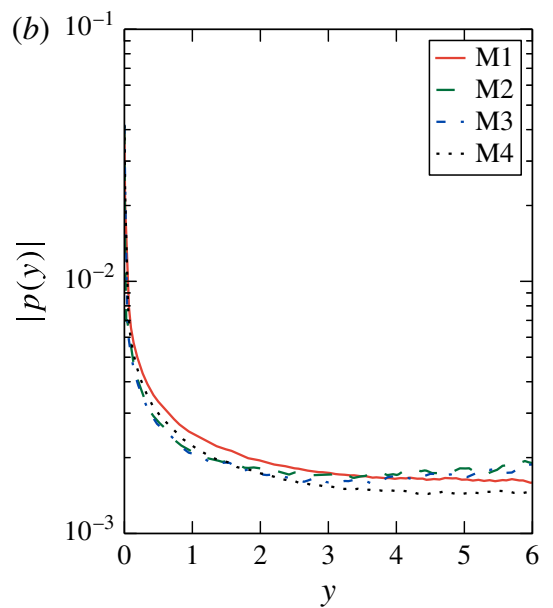

FIGURE 22. (Colour online) (a) Exponential growth in the streamwise direction to comply with the dispersion relation of the advection equation $\omega=\alpha$. (b) Amplitude of pressure fluctuations along a vertical line through the trailing edge.

Following the explanation given in Garnaud et al. (2013) in the context of global modes in compressible jets, this feature is associated with the advection dynamics. It represents features of temporally stable perturbations that were emitted earlier: due to pure advection, they have thus higher amplitudes compared to perturbations that have been emitted at the current time.

\subsection{Free-stream modes}

We have presented above the direct global modes that describe instability mechanisms of the flow in the vicinity of the aerofoil. The description of the operator $\boldsymbol{A}$ in terms of these global modes is, however, incomplete since a representation of the free-stream dynamics is missing.

In figure 23 we display two examples of global modes, labelled F1 and F2, that exhibit free-stream dynamics. The eigenvalues associated with this family of modes have negative temporal growth rates. They are furthermore characterized by a prominent spatial support in the free stream. A noteworthy property is the fact that they show spatial exponential growth towards the outlet and well-defined wavenumbers in the two spatial directions. The features of the free-stream modes are reminiscent of the pseudospectral characteristics of both the advection-diffusion equation and the wave equation with nearly absorbing boundary conditions. The reader is referred to Trefethen (1997) for further details. From a qualitative point of view, the free-stream global modes can be understood as the result of both the finite size of the domain and the dispersion relation of the one-dimensional advection equation $\omega=\alpha$.

Since the domain has a finite extent, any perturbation in the free stream leaves the domain after a convective time period. As a consequence, the global modes that describe the free-stream dynamics are damped. In the light of this fact, it is important to remark that the growth rates of the free-stream modes are inherently dependent on the size of the domain, and in the limit of an infinitely large domain they converge towards neutrality, i.e. a zero temporal growth rate. As before, for a selected $\alpha$ (such that the diffusive effects are negligible), the physics in the 

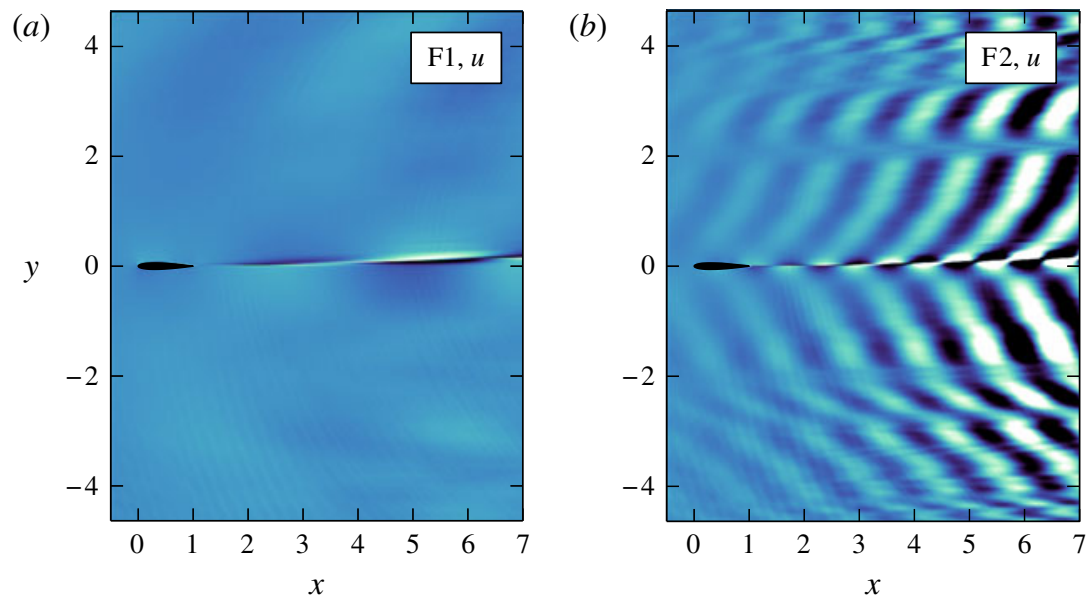

FIGURE 23. (Colour online) Selected free-stream modes: $(a) \omega_{\mathrm{F} 1}=1.11-0.24 \mathrm{i}$ and (b) $\mathrm{F} 2 \omega_{\mathrm{F} 2}=4.97-0.49 \mathrm{i}$.

free stream can be approximated by pure advection, and therefore the free-stream eigenvalues with negative temporal growth rates $\omega$ have associated global modes with wavenumber $\alpha \approx \omega$ : since $\omega_{i}<0$, the free-stream global modes display structures with an associated exponential spatial growth of $-\alpha_{i}>0$. In the global spectrum there is no clear separation between free-stream modes and the global modes presented before. Nonetheless, our calculations show that they are predominant in the region of low temporal growth rates, where the computed eigenvalues have a rather large residual.

\section{Summary and conclusions}

We have addressed the generation of discrete acoustic tones in flow around aerofoils using numerical techniques. The primary purpose of this investigation has been to gain insight into the physical mechanisms underlying the occurrence of multiple peaks in the acoustic spectrum and isolating the principal features of aeroacoustic feedback, from a global point of view. Nonlinear simulations of the compressible Navier-Stokes equations showed good agreement with previous experimental and numerical investigations; in particular, the pressure spectrum displayed strong, equispaced peaks in frequency, typical of the tonal noise phenomenon and widely reported in the literature. Two separation bubbles have been found: on the suction and pressure surfaces of the aerofoil. Based on their dynamics, a quasi-steady state establishes itself, consisting of vortex shedding and intense acoustic scattering at the trailing edge.

Further insight could be gained from an impulse response analysis of the linearized equations, where a localized source of perturbation has been placed near the leading edge of the aerofoil and evolved in time. A cyclical process consisting of (i) wavepackets propagating in the suction-side and pressure-side boundary layer, (ii) their interaction in the vicinity of the trailing edge, (iii) the radiation of sound from the trailing edge, and (iv) the restimulation of the suction-side and pressure-side boundary layer, could be observed and analysed. The time scales and frequencies involved in these processes closely matched the ones observed in the nonlinear simulations. In 
addition, this type of analysis provided clear evidence for a cross-interaction of the pressure-side and suction-side dynamics by means of an acoustic feedback mechanism.

A global stability analysis of the linearized operator revealed a stable spectrum with distinct families of global modes corresponding to various physical processes: the lowfrequency flapping of the separation bubbles, the high-frequency dynamics supported by the shear layers of the separation bubbles, and the free-stream and wake dynamics. These links could be established by associating eigenvalues in the global spectrum with the coherent structures given by the corresponding global modes. The overall dynamics, however, is dominated by the least-stable global modes which exhibit the typical range of acoustic tones. It is worth pointing out that, among all identified modes, only the leading modes had a significant acoustic component associated with their flow fields. Furthermore it has been found that the temporal growth rates of the leading modes follow a peak structure where the frequencies are in remarkably close agreement with the discrete frequencies observed in the nonlinear simulations. The associated spatial structures capture the growth of hydrodynamic instabilities on the suction surface and in the near wake. According to the frequency spacing and the spatial structure, these global modes are associated with the feedback loop observed in both the impulse response and the nonlinear simulations.

\section{Acknowledgements}

This work was performed using HPC resources from GENCI-CINES (grant 2013026451).

\section{Appendix A. Inner product for compressible flows}

The inner product $\langle\cdot, \cdot\rangle$ used in the present study is based on the small-perturbation energy norm derived by Chu (1965) and Hanifi, Schmid \& Henningson (1996) for compressible flows. The energy $\mathscr{E}$ contained in a volume $\mathscr{V}$, written in terms of the variables $(p, s, \boldsymbol{u})$, is given by

$$
2 \mathscr{E}=\int_{\mathscr{V}}\left(\frac{1}{\gamma^{2} M^{2}} \frac{p^{2}}{\bar{p}}+\frac{\gamma-1}{\gamma^{2} M^{2}} \bar{p} s^{2}+\bar{\rho}\|\boldsymbol{u}\|^{2}\right) \mathrm{d} \mathscr{V},
$$

where $\bar{\rho}$ and $\bar{p}$ stand for the base-flow density and pressure, respectively. The dependence on the physical coordinates $\boldsymbol{x}$ has been omitted, and all quantities are non-dimensionalized. Once the previous expression is spatially discretized in the computational domain, the inner product reads

$$
\langle\boldsymbol{w}, \boldsymbol{v}\rangle=\boldsymbol{w}^{H} \boldsymbol{M} \boldsymbol{v}
$$

with

$$
\boldsymbol{M}=\operatorname{diag}\left\{\boldsymbol{m}_{i}\right\}, \quad \text { and } \quad \boldsymbol{m}_{i}=\left(\begin{array}{cccc}
\frac{1}{\gamma^{2} M^{2}} \frac{1}{\bar{p}} & 0 & 0 & 0 \\
0 & \frac{\gamma-1}{\gamma^{2} M^{2}} \bar{p} & 0 & 0 \\
0 & 0 & \bar{\rho} & 0 \\
0 & 0 & \bar{\rho}
\end{array}\right)_{i}\left|\operatorname{det}\left(\frac{\partial \boldsymbol{x}}{\partial \boldsymbol{\xi}}\right)\right|_{i} \Delta \xi_{i} \Delta \eta_{i}
$$

where the subscript $i$ denotes the grid points. 


\section{REFERENCES}

ADAMS, N. A. \& SHARIFF, K. 1996 A high-resolution hybrid compact-ENO scheme for shockturbulence interaction problems. J. Comput. Phys. 127 (1), 27-51.

Akbar, T. 2010 Simulation of three-dimensional flows past infinite cylindrical bodies. PhD thesis, Université de Strasbourg.

Alizard, F., Cherubini, S. \& Robinet, J.-C. 2009 Sensitivity and optimal forcing response in separated boundary layer flows. Phys. Fluids 21, 064108.

ARbey, H. \& BAtAille, J. 1983 Noise generated by aerofoil profiles placed in a uniform laminar flow. J. Fluid Mech. 134, 33-47.

Arcondoulis, E., Doolan, C. J. \& Zander, A. C. 2009 Aerofoil noise measurements at various angles of attack and low Reynolds number. In Proceedings of ACOUSTICS 2009 Adelaide, 23rd-25th November 2009. Australian Acoustics Society.

BARKLEY, D. 2006 Linear analysis of the cylinder wake mean flow. Europhys. Lett. 75 (5), 750-756.

Bodony, D. J. 2006 Analysis of sponge zones for computational fluid mechanics. J. Comput. Phys. 212 (2), 681-702.

Brooks, T. F., Pope, D. S. \& Marcolini, M. A. 1989 Aerofoil self-noise and prediction. NASA Ref. Pub. 1218.

Cherubini, S., Robinet, J.-Ch. \& De Palma, P. 2010 The effects of non-normality and nonlinearity of the Navier-Stokes operator on the dynamics of a large laminar separation bubble. Phys. Fluids 22, 014102.

Chong, T. P. \& Joseph, P. 2012 'Ladder' structure in tonal noise generated by laminar flow around an aerofoil. J. Acoust. Soc. Am. 131 (6), 461-467.

CHU, B. T. 1965 On the energy transfer to small disturbances in fluid flow (Part I). Acta Mechanica 1 (3), 215-234.

Desquesnes, G., Terracol, M. \& Sagaut, P. 2007 Numerical investigation of the tone noise mechanism over laminar aerofoils. J. Fluid Mech. 591, 155-182.

Dovgal, A. V., Kozlov, V. V. \& Michalke, A. 1994 Laminar boundary layer separation: instability and associated phenomena. Prog. Aerosp. Sci. 30 (1), 61-94.

Edwards, W. S., Tuckerman, L. S., Friesner, R. A.\& Sorensen, D. C. 1994 Krylov methods for the incompressible Navier-Stokes equations. J. Comput. Phys. 110 (1), 82-102.

Ehrenstein, U. \& Gallaire, F. 2008 Two-dimensional global low-frequency oscillations in a separating boundary-layer flow. J. Fluid Mech. 614, 315-327.

FINK, M. R. 1975 Prediction of aerofoil tone frequencies. J. Aircraft 12 (2), 118-120.

Fosas De Pando, M., SiPP, D. \& SChMid, P. J. 2012 Efficient evaluation of the direct and adjoint linearized dynamics from compressible flow solvers. J. Comput. Phys. 231 (23), 7739-7755.

Gallaire, F., Marquillie, M. \& Ehrenstein, U. 2007 Three-dimensional transverse instabilities in detached boundary layers. J. Fluid Mech. 571, 221-233.

Garnaud, X., Lesshafft, L., Schmid, P. J. \& Huerre, P. 2013 Modal and transient dynamics of jet flows. Phys. Fluids 044103 .

Le Garrec, T., Gloerfelt, X. \& Corre, C. 2008 A numerical insight into the effect of confinement on trailing edge noise. J. Acoust. Soc. Am. 123 (5), 3022.

HAMmOND, D. A. \& REDEKOPP, L. G. 1998 Local and global instability properties of separation bubbles. Eur. J. Mech. (B/Fluids) 17 (2), 145-164.

Hanifi, A., Schmid, P. J. \& Henningson, D. S. 1996 Transient growth in compressible boundary layer flow. Phys. Fluids 8, 826-837.

HernándeZ, V., RomÁn, J. E. \& VIDAL, V. 2005 SLEPc: a scalable and flexible toolkit for the solution of eigenvalue problems. ACM Trans. Math. Softw. 31 (3), 351-362.

Jones, L. E. \& SANDBERG, R. D. 2011 Numerical analysis of tonal aerofoil self-noise and acoustic feedback-loops. J. Sound Vib. 330, 6137-6152.

Jones, L. E., SAndberg, R. D. \& SAndham, N. D. 2008 Direct numerical simulations of forced and unforced separation bubbles on an aerofoil at incidence. J. Fluid Mech. 602, 175-207.

Jones, L. E., SAndberg, R. D. \& SAndham, N. D. 2010 Stability and receptivity characteristics of a laminar separation bubble on an aerofoil. J. Fluid Mech. 648, 257-296. 
Kennedy, C. A., Carpenter, M. H. \& Lewis, R. M. 2000 Low-storage, explicit Runge-Kutta schemes for the compressible Navier-Stokes equations. Appl. Numer. Maths 35 (3), 177-219.

Kingan, M. J. \& Pearse, J. R. 2009 Laminar boundary layer instability noise produced by an aerofoil. J. Sound Vib. 322 (4-5), 808-828.

LELE, S. K. 1992 Compact finite difference schemes with spectral-like resolution. J. Comput. Phys. 103 (1), 16-42.

Lodato, G., Domingo, P. \& Vervisch, L. 2008 Three-dimensional boundary conditions for direct and large-eddy simulation of compressible viscous flows. J. Comput. Phys. 227 (10), 5105-5143.

Longhouse, R. E. 1977 Vortex shedding noise of low tip speed, axial flow fans. J. Sound Vib. 53 (1), 25-46.

Marquet, O., Lombardi, M., Chomaz, J.-M., Sipp, D. \& Jacquin, L. 2009 Direct and adjoint global modes of a recirculation bubble: lift-up and convective non-normalities. J. Fluid Mech. 622, $1-21$.

Marquet, O., Sipp, D., Chomaz, J.-M. \& Jacquin, L. 2008 Amplifier and resonator dynamics of a low-Reynolds-number recirculation bubble in a global framework. J. Fluid Mech. 605, 429-443.

McAlpine, A., Nash, E. C. \& Lowson, M. V. 1999 On the generation of discrete frequency tones by the flow around an aerofoil. J. Sound Vib. 222 (5), 753-779.

Nakano, T., Fujisawa, N. \& LeE, S. 2006 Measurement of tonal-noise characteristics and periodic flow structure around NACA0018 aerofoil. Exp. Fluids 40 (3), 482-490.

Nash, E. C., Lowson, M. V. \& MCAlpine, A. 1999 Boundary-layer instability noise on aerofoils. J. Fluid Mech. 382, 27-61.

Paterson, R. W., Vogt, P. G., Fink, M. R. \& Much, C. L. 1973 Vortex noise of isolated aerofoils. J. Aircraft 10 (5), 296-302.

Poinsot, T. J. \& LELE, S. K. 1992 Boundary conditions for direct simulations of compressible viscous flows. J. Comput. Phys. 101 (1), 104-129.

SandberG, R. D., Jones, L. E., Sandham, N. D. \& Joseph, P. F. 2009 Direct numerical simulations of tonal noise generated by laminar flow past aerofoils. J. Sound Vib. 320, 838-858.

Schmid, P. J. \& Henningson, D. S. 2002 On the stability of a falling liquid curtain. J. Fluid Mech. 463, 163-171.

SesterhenN, J. 2000 A characteristic-type formulation of the Navier-Stokes equations for high order upwind schemes. Comput. Fluids 30 (1), 37-67.

SIDJE, R. B. 1998 EXPOKIT: a software package for computing matrix exponentials. ACM Trans. Math. Softw. 24 (1), 130-156.

Stewart, G. W. 2002 A Krylov-Schur algorithm for large eigenproblems. SIAM J. Matrix Anal. Applics. 23 (3), 601-614.

TAkagi, Y., Fujisawa, N., Nakano, T. \& Nashimoto, A. 2006 Cylinder wake influence on the tonal noise and aerodynamic characteristics of a NACA0018 aerofoil. J. Sound Vib. 297 (3-5), $563-577$.

TAM, C. K. W. 1974 Discrete tones of isolated aerofoils. J. Acoust. Soc. Am. 55 (6), 1173-1177.

TAM, C. K. W. \& JU, H. 2011 Aerofoil tones at moderate Reynolds number. J. Fluid Mech. 690, 536-570.

Trefethen, L. N. 1997 Pseudospectra of linear operators. SIAM Rev. 39 (3), 383-406.

Wright, S. E. 1976 The acoustic spectrum of axial flow machines. J. Sound Vib. 45 (2), 165-223. 